\title{
Chebyshev Collocation Method for the Free Vibration Analysis of Geometrically Exact Beams with Fully Intrinsic Formulation
}

\author{
Pedram Khaneh Masjedi ${ }^{\mathrm{a}, *}$, Alireza Maheri ${ }^{\mathrm{b}}$, \\ ${ }^{a}$ Department of Aerospace Engineering, Amirkabir University of Technology, Tehran, Iran \\ ${ }^{b}$ School of Engineering, University of Aberdeen, Aberdeen, UK
}

\begin{abstract}
A Chebyshev collocation method is presented for the free vibration analysis of geometrically exact nonlinear beams with fully intrinsic formulation. The intrinsic formulation of the governing equations of the beam contains neither displacement nor rotation variables. The proposed collocation discretization technique is based on the Chebyshev points as the collocation points and the orthogonal Chebyshev polynomials as the trial functions. This method is successfully applied to the eigenvalue analysis of the linearized intrinsic governing equations of a nonlinear beam. A number of test cases have been considered for either straight or pretwisted beams and the obtained results are compared to the analytical, numerical as well as experimental results. In order to show the applicability of current approach for real-life engineering problems, a composite wind turbine rotor blade with non-uniform distribution of properties is also considered. In all test cases a very good concordance has been observed. The proposed method bypasses the integrations common in finite element based methods and difficulties associated with finite rotations interpolation and while exhibiting a very good accuracy compared to the finite element results, it is computationally more efficient and simpler to implement in a computer programming code.
\end{abstract}

Keywords: Chebyshev Collocation Method, Geometrically Exact Beam, Intrinsic Formulation, Free Vibration, Pretwisted Beam, Composite Rotor Blade, Composite Beam

\section{Introduction}

In the context of the mathematical modeling of geometrically nonlinear beams the so called geometrically exact theories have attracted a great deal of attentions so far. In the geometrically exact beam theories no specific assumptions are made with regards to the displacement field of the beam and the finite rotations are utilized for the kinematical description of the beam deformations which make these kinds of beam theories, among other theories, the most versatile and the most loyal to the true nature of the beam problem. The pioneering work of Reissner (1973) on the statics of geometrically exact beams has been the source of

\footnotetext{
* Corresponding author: Phone:+98 9122603502

Email addresses: p_masjedi@aut.ac.ir (Pedram Khaneh Masjedi), alireza.maheri@abdn.ac.uk (Alireza Maheri ) 
many future extensions and generalizations of geometrically exact beam theories. Simo (1985) has extended the Reissner's model to the dynamic case. Cardona and Géradin (1988) have presented a dynamic finite element beam model considering large finite rotations and have used an updated Lagrangian approach for the numerical analysis. Iura and Atluri (1988) have used similar assumptions to those made by Reissner (1973) and Simo (1985) and have proposed a model for the dynamic analysis of space beams. Based on the generalized strain concept represented by Reissner (1973), Hodges (1990) has presented a set of intrinsic equations of motion for moving initially curved and twisted beams. Many authors have developed more or less similar models for the dynamics of geometrically exact beams, e.g. see Simo and Vu-Quoc (1988, 1991), Pai and Nayfeh (1994), Petrov and Géradin (1998), Ibrahimbegović and Mikdad (1998), Jelenić and Crisfield (1999), Romero and Armero (2002), Mäkinen (2007) and many others. However a full bibliography in this context is out of the scope of the current work.

A vast majority of the numerical treatments of the geometrically exact beams has been devoted to the finite element methods (FEM) which are mostly based on the interpolation of finite rotations. The interpolation and parametrization of finite rotations generally involve important issues such as objectivity of the strain field, singularities and orthogonality of the finite rotations field which cause difficulties in various approaches that are used for the interpolation of finite rotations. In order to avoid the difficulties associated with the interpolation of finite rotations, the formulations with no rotational degrees of freedom have been emerged. For a more thorough review of various approaches with rotation free formulation the readers are referred to Khaneh Masjedi and Ovesy (2015).

One approach that is free from rotational variables is to consider the intrinsic governing equations of motion. The intrinsic equations of motion are the linear and angular momentum balances and in general they are free from any displacement or rotational variables. The most attractive characteristic of intrinsic formulation is the low order of nonlinearity which is quadratic at most and as a result makes it desirable for numerical analysis.

Hegemier and Nair (1977) have presented an intrinsic formulation for the dynamics of anisotropic pretwisted beams but they did not present any numerical results. Hodges (2003) has introduced a set of complete intrinsic equations for the dynamics of initially curved and twisted geometrically exact beams and has shown the advantages of fully intrinsic formulation for a beam under non-conservative transverse follower force. The latter approach which is based on a finite difference scheme has been later used by Chang and Hodges (2009a) and Chang and Hodges (2009b) for the free vibration and stability analysis of curved beams. Khouli et al. (2009) and Ghorashi and Nitzsche (2009) also have used finite difference schemes for the spatial discretization of the intrinsic formulation presented by Hodges (2003) for the dynamic analysis of helicopter rotor blades. Patil and Althoff (2011) have proposed a Galerkin's method in which the Legendre polynomials are used as trial functions for the dynamic and free vibration analysis of fully intrinsic beam formulation. This approach has been extended further through the implementation of a variable order finite 
element by Patil and Hodges (2011).

Collocation methods exhibit superior computational efficiency and accuracy compared to Galerkin's and finite element methods. Schillinger et al. (2013) have shown that the collocation method significantly reduces the computational cost in comparison to Galerkin's and finite element methods and also can be orders of magnitude faster than these methods to achieve a specified level of accuracy. Khaneh Masjedi and Ovesy (2015) have introduced the Chebyshev collocation method to the static analysis of geometrically exact beams with intrinsic formulation and have shown that this scheme while computationally efficient exhibits comparable accuracy as distinct from other numerical schemes such as finite element method.

The main objectives of the current work have been to extend the approach presented by Khaneh Masjedi and Ovesy (2015) for the first time to the free vibration analysis of geometrically exact beams with intrinsic formulation and to show the accuracy and applicability of this scheme for the proper treatment of eigenvalue analysis of geometrically exact beams with fully intrinsic formulation. Additionally the free vibration problem for various boundary conditions in the context of geometrically exact beams with intrinsic formulation is considered which is addressed very rarely in the literature. It will be shown in this paper that the proposed method of analysis is applicable to real life engineering problems with nonuniform distribution of properties and spatial initial twist and curvature as well as classical benchmark problems. The application of this method can be further extended to more complex cases, which require several beam assemblage, by adopting hybrid approaches; for instance, with Strong Formulation Finite Element Method (Tornabene et al. (2015)).

The content of the current paper is outlined as follows. In Section 2 a description of the intrinsic equations of motion of a geometrically exact beam is given. These equations will be linearized about a steady state position using perturbation method. It should be noted that for the current work in order to be self-sufficient some parts in theoretical developments are borrowed from Khaneh Masjedi and Ovesy (2015). In Section 3 the Chebyshev collocation method is introduced into the linearized intrinsic formulation of the beam and a general eigenvalue problem is constructed. In Section 4 illustrating examples are presented based on the proposed approach and the obtained results are compared to the analytical as well as other numerical results. It is shown that the current approach while computationally efficient and easy to implement, exhibits comparable accuracy to the other numerical schemes such as Galerkin's or finite element method. Finally in Section 5 some concluding remarks are drawn.

\section{Geometrically Exact Beam Governing Equations}

\subsection{Beam Kinematics}

The geometry of an initially curved and twisted beam is depicted for the deformed and undeformed configurations in Fig. 1 The base vectors $\mathbf{I}_{1}, \mathbf{I}_{2}$ and $\mathbf{I}_{3}$ are considered as the inertial reference frame; since 
all motions are measured and described relative to this frame, the base vectors $\mathbf{e}_{1}, \mathbf{e}_{2}$ and $\mathbf{e}_{3}$ are attached to the reference line in the undeformed state such that $\mathbf{e}_{1}$ is always tangent to the beam reference line (Beam longitudinal axis). At each position on the reference line the base vectors $\mathbf{e}_{2}$ and $\mathbf{e}_{3}$ comprise the cross-sectional plane of the beam. The base vectors $\mathbf{e}_{1}^{*}, \mathbf{e}_{2}^{*}$ and $\mathbf{e}_{3}^{*}$ which constitute an orthonormal set are expressed in the deformed state of the beam and are considered as the deformed frame. The base vectors $\mathbf{e}_{2}^{*}$, $\mathbf{e}_{3}^{*}$ could be considered as the rotated and translated base vectors $\mathbf{e}_{2}$ and $\mathbf{e}_{3}$ related to the cross-sectional plane in the undeformed state and they are in the plane of the cross-section in the deformed state. It is assumed that the cross-section of the beam can undergo shear deformations so generally speaking the base vector $\mathbf{e}_{1}^{*}$ is not necessarily tangent to the longitudinal axis of the deformed beam.

The 1D generalized strain measures for an initially curved and twisted beam can be expressed as Khaneh Masjedi and Ovesy (2015)):

$$
\begin{gathered}
\boldsymbol{\gamma}=\boldsymbol{\Lambda}^{T} \cdot \mathbf{R}_{0,1}-\mathbf{r}_{0,1} \\
\boldsymbol{\kappa}=\boldsymbol{\Lambda}^{T} . \mathbf{K}-\mathbf{k}
\end{gathered}
$$

in which; $\boldsymbol{\Lambda}$ is a linear transformation that relates the orthonormal base vectors $\mathbf{e}_{i}$ and $\mathbf{I}_{i}$ and is only function of the beam reference line (i.e. $x_{1}$ ). The vectors $\mathbf{k}$ and $\mathbf{K}$ are the curvature vectors of the beam in the undeformed and deformed state of the beam. In the matrix form we have; $k=\left[k_{1}, k_{2}, k_{3}\right]^{T}$ and $K=\left[K_{1}, K_{2}, K_{3}\right]^{T}$. When $\mathbf{k}$ is expressed in the undeformed frame (i.e. $\mathbf{e}_{i}$ ) and $\mathbf{K}$ is expressed in the deformed frame (i.e. $\mathbf{e}_{i}^{*}$ ), $k_{1}$ is regarded as the initial twist and $k_{2}$ and $k_{3}$ are regarded as the initial curvatures of the beam and $K_{1}$ is the twist and $K_{2}$ and $K_{3}$ are the curvatures of the beam in the deformed state, $\mathbf{R}_{0,1}=\partial \mathbf{R}_{0} / \partial x_{1}$ and $\mathbf{r}_{0,1}=\partial \mathbf{r}_{0} / \partial x_{1} . \mathbf{r}_{0}$ and $\mathbf{R}_{0}$ are the position vectors of the beam reference line in the undeformed and deformed configurations respectively and we have; $\mathbf{R}_{0}=\mathbf{r}_{0}+\mathbf{u}_{0}$, where $\mathbf{u}_{0}$ is the displacement vector of the beam reference line. It should be noted that the dot operator is used throughout the paper for product between any two vectors and/or matrices.

The above strain measures are called force and moment strain (Reissner (1973); Hodges (1990)) since they are conjugate to the cross-sectional forces and moments. These generalized strain measures can be expressed in the matrix form as; $\gamma=\left[\gamma_{11}, 2 \gamma_{12}, 2 \gamma_{13}\right]^{T}$ and $\kappa=\left[\kappa_{1}, \kappa_{2}, \kappa_{3}\right]^{T}$. Based on the Frenet-Serret formula the derivative of the base vectors $\mathbf{e}_{i}$ and $\mathbf{e}_{i}^{*}$ with respect to the beam reference line (i.e. $\left.x_{1}\right)$ can be obtained as:

$$
\begin{gathered}
\left(\mathbf{e}_{i}\right)_{, 1}=\frac{\partial \mathbf{e}_{i}}{\partial x_{1}}=\tilde{\mathbf{k}} \cdot \mathbf{e}_{i} \\
\left(\mathbf{e}_{i}^{*}\right)_{, 1}=\frac{\partial \mathbf{e}_{i}^{*}}{\partial x_{1}}=\tilde{\mathbf{K}} \cdot \mathbf{e}_{i}^{*}
\end{gathered}
$$




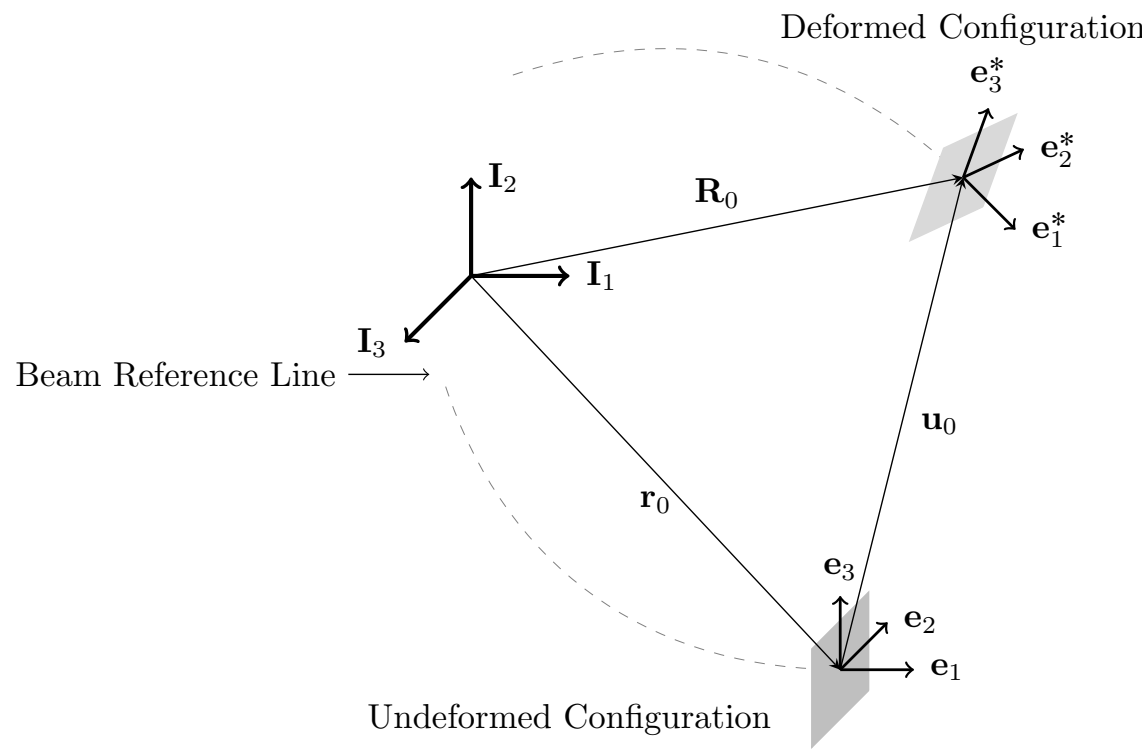

Figure 1: Initially Curved and Twisted Beam in the Undeformed and Deformed Configurations

Similarly one can write for the derivative of the base vectors $\mathbf{e}_{i}^{*}$ with respect to time as follows;

$$
\left(\dot{\mathbf{e}}_{i}^{*}\right)=\frac{\partial \mathbf{e}_{i}^{*}}{\partial t}=\widetilde{\Omega} \cdot \mathbf{e}_{i}^{*}
$$

In which $\boldsymbol{\Omega}$ is considered as the angular velocity vector of the deformed base vectors $\mathbf{e}_{i}^{*}$. It should be noted that $(\sim)$ is the cross-product operator.

\subsection{Extended Hamilton's Principle}

Using the extended Hamilton's principle the intrinsic equations of motion can be derived. The extended Hamilton's principle is expressed as:

$$
\int_{t_{1}}^{t_{2}} \int_{0}^{L}\left[\delta \mathcal{T}-\delta \mathcal{U}+\overline{\delta \mathcal{W}}_{e x t}\right] d x_{1} d t=0
$$

where $\delta \mathcal{U}$ is the virtual internal work or strain energy, $\delta \mathcal{T}$ is the virtual kinetic energy and $\overline{\delta \mathcal{W}}_{\text {ext }}$ is the virtual work of external applied loads, per unit length of the beam.

The virtual strain energy per unit length of the beam can be expressed as (Khaneh Masjedi and Ovesy (2015)):

$$
\delta \mathcal{U}=\mathbf{F} . \delta \boldsymbol{\Gamma}+\mathbf{M} . \delta \mathcal{K}
$$

in which $\mathbf{F}$ and $\mathbf{M}$ are internal force and moment and are defined as:

$$
\mathbf{F}=\frac{\partial \mathcal{U}}{\partial \boldsymbol{\Gamma}}, \quad \mathbf{M}=\frac{\partial \mathcal{U}}{\partial \mathcal{K}}
$$


where $\mathbf{F}=\boldsymbol{\Lambda} . \mathbf{f}, \mathbf{M}=\boldsymbol{\Lambda} . \mathbf{m}, \delta \boldsymbol{\Gamma}=\boldsymbol{\Lambda} . \delta \gamma$ and $\delta \mathcal{K}=\boldsymbol{\Lambda} . \delta \boldsymbol{\kappa}$. Herein $\boldsymbol{\Lambda}$ acts as a push-forward operator and as a result if $\mathbf{f}, \mathbf{m}, \delta \boldsymbol{\gamma}$ and $\delta \boldsymbol{\kappa}$ are expressed in the undeformed frame, $\mathbf{F}, \mathbf{M}, \delta \mathbf{\Gamma}$ and $\delta \mathcal{K}$ are expressed in the deformed frame. The virtual strain energy of the beam can be expressed as (Khaneh Masjedi and Ovesy (2015)):

$$
\begin{gathered}
\int_{0}^{L} \delta \mathcal{U} d x_{1}= \\
-\int_{0}^{L}\left[\left(\mathbf{F},_{1}+\widetilde{\mathbf{K}} \cdot \mathbf{F}\right) \cdot \delta \mathbf{R}_{0}+\right. \\
\left.\left(\widetilde{\mathbf{R}}_{0,1} \cdot \mathbf{F}+\mathbf{M}_{, 1}+\widetilde{\mathbf{K}} \cdot \mathbf{M}\right) \cdot \delta \phi\right] d x_{1}+ \\
\mathbf{F} .\left.\delta \mathbf{R}_{0}\right|_{0} ^{L}+\left.\mathbf{M} \cdot \delta \phi\right|_{0} ^{L}
\end{gathered}
$$

The virtual kinetic energy per unit length of the beam can be expressed as:

$$
\delta \mathcal{T}=\mathbf{L} . \delta \boldsymbol{V}^{*}+\mathbf{P} . \delta \mathbf{\Omega}^{*}
$$

in which $\mathbf{L}$ and $\mathbf{P}$ are linear and angular momentum and are defined as:

$$
\mathbf{L}=\frac{\partial \mathcal{T}}{\partial \boldsymbol{V}}, \quad \mathbf{P}=\frac{\partial \mathcal{T}}{\partial \boldsymbol{\Omega}}
$$

In Eq. (2.10) $\delta \boldsymbol{V}^{*}$ and $\delta \boldsymbol{\Omega}^{*}$ are conjugate variation of linear and angular velocities to linear and angular momentum respectively and we have; $\delta \boldsymbol{V}^{*}=\boldsymbol{\Lambda} . \delta \boldsymbol{v}, \delta \boldsymbol{\Omega}^{*}=\boldsymbol{\Lambda} . \delta \boldsymbol{\omega}, \mathbf{L}=\boldsymbol{\Lambda} . \mathbf{l}$ and $\mathbf{P}=\boldsymbol{\Lambda} . \mathbf{p}$.

One can write:

$$
\begin{gathered}
\delta \boldsymbol{v}=\boldsymbol{\Lambda}^{T} \cdot\left(\widetilde{\delta \phi}^{T} \cdot \dot{\mathbf{R}_{\mathbf{0}}}+\delta \dot{\mathbf{R}_{\mathbf{0}}}\right) \\
\delta \boldsymbol{\omega}=\boldsymbol{\Lambda}^{T} . \delta \dot{\boldsymbol{\phi}}
\end{gathered}
$$

From Eqs.2.10, 2.12 and 2.13 the kinetic energy of the beam is expressed as:

$$
\begin{aligned}
& \int_{t_{1}}^{t_{2}} \delta \mathcal{T} d t= \\
& \int_{t_{1}}^{t_{2}}\left[(\boldsymbol{\Lambda} \cdot \mathbf{l}) \cdot\left(\delta \dot{\mathbf{R}}_{0}+\dot{\widetilde{\mathbf{R}}}_{0} \cdot \delta \boldsymbol{\phi}\right)+(\boldsymbol{\Lambda} \cdot \mathbf{p}) \cdot \delta \dot{\phi}\right] d t= \\
& -\int_{t_{1}}^{t_{2}}\left[(\dot{\bar{\Lambda} \cdot \mathbf{l}}) \cdot \delta \mathbf{R}_{0}+\left(\dot{\widetilde{\mathbf{R}}}_{0} \cdot(\boldsymbol{\Lambda} \cdot \mathbf{l})+(\dot{\boldsymbol{\Lambda} \cdot \mathbf{p}})\right) \cdot \delta \phi\right] d t \\
& \quad+(\boldsymbol{\Lambda} . \mathbf{l}) .\left.\delta \mathbf{R}_{0}\right|_{t_{1}} ^{t_{2}}+(\boldsymbol{\Lambda} \cdot \mathbf{p}) .\left.\delta \phi\right|_{t_{1}} ^{t_{2}}
\end{aligned}
$$

further expanding $(\dot{\overline{\Lambda . l}})$ and $(\dot{\overline{\boldsymbol{\Lambda} . \mathbf{p}}})$;

$$
\begin{aligned}
& (\dot{\overline{\Lambda .}})=\dot{\Lambda} .1+\Lambda . \dot{l} \\
& =\dot{\Lambda} .\left(\boldsymbol{\Lambda}^{T} \cdot \boldsymbol{\Lambda}\right) . \mathbf{l}+\boldsymbol{\Lambda} . \dot{\mathbf{l}} \\
& =\widetilde{\mathbf{\Omega}} .(\boldsymbol{\Lambda} . \mathbf{l})+\boldsymbol{\Lambda} . \dot{\mathrm{i}} \\
& =\tilde{\mathbf{\Omega}} \cdot \mathbf{L}+\dot{\mathbf{L}}
\end{aligned}
$$


similarly;

$$
\begin{aligned}
\left(\frac{\dot{\boldsymbol{\Lambda}} \mathbf{p}}{\mathbf{p}}\right) & =\widetilde{\mathbf{\Omega}} \cdot(\boldsymbol{\Lambda} \cdot \mathbf{p})+\mathbf{\Lambda} \cdot \dot{\mathbf{p}} \\
& =\widetilde{\mathbf{\Omega}} \cdot \mathbf{P}+\dot{\mathbf{P}}
\end{aligned}
$$

Introducing Eqs. 2.15) and 2.16) into Eq. 2.14) will lead to:

$$
\begin{aligned}
& \quad \int_{t_{1}}^{t_{2}} \delta \mathcal{T} d t= \\
& -\int_{t_{1}}^{t_{2}}\left[(\widetilde{\boldsymbol{\Omega}} \cdot \mathbf{L}+\dot{\mathbf{L}}) \cdot \delta \mathbf{R}_{0}+\left(\dot{\widetilde{\mathbf{R}}}_{0} \cdot \mathbf{L}+\widetilde{\mathbf{\Omega}} \cdot \mathbf{P}+\dot{\mathbf{P}}\right) \cdot \delta \phi\right] d t \\
& \quad+\mathbf{L} .\left.\delta \mathbf{R}_{0}\right|_{t_{1}} ^{t_{2}}+\mathbf{P} .\left.\delta \phi\right|_{t_{1}} ^{t_{2}}
\end{aligned}
$$

The virtual work of the external applied forces i.e. $\overline{\mathbf{f}}$ and applied moments i.e. $\overline{\mathbf{m}}$ are given by;

$$
\int_{t_{1}}^{t_{2}} \int_{0}^{L} \overline{\delta \mathcal{W}}_{e x t} d x_{1}=\int_{t_{1}}^{t_{2}} \int_{0}^{L}\left[\overline{\mathbf{f}} . \delta \mathbf{R}_{0}+\overline{\mathbf{m}} . \delta \phi\right] d x_{1}
$$

From Eqs. 2.6 2.9), 2.17) and 2.18), the intrinsic equations of motion of a spatial beam are derived as follows;

$$
\begin{gathered}
\mathbf{F}_{, 1}+\tilde{\mathbf{K}} \cdot \mathbf{F}+\overline{\mathbf{f}}=\dot{\mathbf{L}}+\widetilde{\mathbf{\Omega}} . \mathbf{L} \\
\mathbf{M}_{, 1}+\widetilde{\mathbf{K}} \cdot \mathbf{M}+\widetilde{\mathbf{R}}_{0,1} \cdot \mathbf{F}+\overline{\mathbf{m}}=\dot{\mathbf{P}}+\widetilde{\mathbf{\Omega}} \cdot \mathbf{P}+\tilde{\mathbf{V}} \cdot \mathbf{L}
\end{gathered}
$$

where $\mathbf{V}=\dot{\mathbf{R}}_{0}$. It is noted that the equations of motion presented in Eq. 2.19] are identical to those of Hodges (1990, 2003).

In order to close the formulation a set of kinematical relations are required. These kinematical relations are presented by Hodges (2003) and are expressed as:

$$
\begin{aligned}
& \dot{\gamma}=\mathbf{V}_{, 1}+\tilde{\mathbf{K}} \cdot \mathbf{V}+\widetilde{\mathbf{R}}_{0,1} \cdot \boldsymbol{\Omega} \\
& \dot{\boldsymbol{\kappa}}=\boldsymbol{\Omega}_{, 1}+\tilde{\mathbf{K}} . \boldsymbol{\Omega}
\end{aligned}
$$

It is noted that $\mathbf{R}_{0,1}$ and $\boldsymbol{\kappa}$ are given by Khaneh Masjedi and Ovesy (2015) as:

$$
\begin{aligned}
& \mathbf{R}_{0,1}=\left(1+\gamma_{11}\right) \mathbf{e}_{1}^{*}+2 \gamma_{1 \alpha} \mathbf{e}_{\alpha}^{*} \\
& \kappa_{i} \mathbf{e}_{i}^{*}=\left(K_{i}-k_{i}\right) \mathbf{e}_{i}^{*}
\end{aligned}
$$

where $\alpha=2,3$ and $i=1,2,3$.

Introducing the linear constitutive equation and the generalized momentum-velocity relations;

$$
\begin{gathered}
\left\{\begin{array}{l}
\boldsymbol{\gamma} \\
\boldsymbol{\kappa}
\end{array}\right\}=\left[\begin{array}{cc}
R & S \\
S^{T} & T
\end{array}\right]\left\{\begin{array}{c}
\mathbf{F} \\
\mathbf{M}
\end{array}\right\} \\
\left\{\begin{array}{l}
\boldsymbol{L} \\
\boldsymbol{P}
\end{array}\right\}=\left[\begin{array}{cc}
\mu \Delta & -\mu \tilde{\bar{x}} \\
\mu \tilde{\bar{x}} & I
\end{array}\right]\left\{\begin{array}{l}
\mathbf{V} \\
\boldsymbol{\Omega}
\end{array}\right\}
\end{gathered}
$$


one can eliminate $\boldsymbol{\gamma}, \boldsymbol{\kappa}, \mathbf{L}$ and $\mathbf{P}$ and only $\mathbf{F}, \mathbf{M}, \mathbf{V}$ and $\boldsymbol{\Omega}$ will remain as unknowns.

In Eq. 2.22 $R, S$ and $T$ are the matrices of cross-sectional flexibility and in Eq. (2.23) $\mu$ is mass per unit length of the beam, $\Delta$ is the identity matrix, $\bar{x}$ is the offset from the reference line of the cross-sectional mass centroid and $\bar{x}=\left[0, \bar{x}_{2}, \bar{x}_{3}\right]^{T}$ and $I$ is the beam cross-section mass moment of inertia per unit length of the beam. Since the presented method is not limited by constitutive equations, beams made of anisotropic materials with arbitrary cross-sectional shapes can be also modelled and analysed using this method. In order to obtain the stiffness matrix of composite beams with arbitrary complex cross-sections a number of finite element based discretization tools have been developed, e.g. see Morandini et al. (2010), Yu et al. (2012) and Genoese et al. (2014) in which Saint-Venant effect (including in-plane and out-of-plane sectional warping deformation) are incorporated. The stiffness matrix obtained from any of such tools can be directly used as the constitutive equation in current beam model. It is also possible to further extend governing equations 2.19) to incorporate the end effect associated with the torsional deformation well-known as Vlasov effect, e.g. see Khouli et al. (2010) for the static case. However it is required to obtain appropriate crosssectional stiffness matrix in which Vlasov effect is included such as works by Yu et al. (2005), Kim et al. (2008) and Kim and Kim (2013). For the case of an isotropic beam in which the beam reference line coincides with the locus of both cross-sectional shear and area centroids, the constitutive equations (Eq. 2.22) are expressed as:

$$
\left\{\begin{array}{c}
\gamma_{11} \\
2 \gamma_{12} \\
2 \gamma_{13} \\
\kappa_{1} \\
\kappa_{2} \\
\kappa_{3}
\end{array}\right\}=\left[\begin{array}{cccccc}
\frac{1}{E A} & 0 & 0 & 0 & 0 & 0 \\
0 & \frac{1}{G A_{2}} & 0 & 0 & 0 & 0 \\
0 & 0 & \frac{1}{G A_{3}} & 0 & 0 & 0 \\
0 & 0 & 0 & \frac{1}{G J} & 0 & 0 \\
0 & 0 & 0 & 0 & \frac{1}{E I_{2}} & 0 \\
0 & 0 & 0 & 0 & 0 & \frac{1}{E I_{3}}
\end{array}\right]\left\{\begin{array}{c}
F_{1} \\
F_{2} \\
F_{3} \\
M_{1} \\
M_{2} \\
M_{3}
\end{array}\right\}
$$

where, $E$ is the elastic modulus, $G$ is the shear modulus defined as $G=E / 2(1+\nu)$ where $\nu$ is the Poisson's ratio, $A$ is the cross-sectional area, $A_{2}=c_{2} A$ and $A_{3}=c_{3} A$ in which $c_{2}$ and $c_{3}$ are the shear correction factors, $I_{2}$ and $I_{3}$ are the cross-sectional moments of inertia and $J$ is the polar moment of inertia. It is noted that the analytical formulations for $c_{2}$ and $c_{3}$ for rectangular cross-sections that is used throughout this work is taken from Hodges (2006), as follows;

$$
\begin{gathered}
c_{2}=\left[\frac{6}{5}+\left(\frac{\nu}{1+\nu}\right)^{2}\left(\frac{a}{b}\right)^{-4}\left(\frac{1}{5}-\frac{18}{\left(\frac{a}{b}\right) \pi^{5}}\right) \sum_{m=1}^{\infty}\left(\frac{\tanh \left(m \pi\left(\frac{a}{b}\right)\right)}{m^{5}}\right)\right]^{-1} \\
c_{3}=\left[\frac{6}{5}+\left(\frac{\nu}{1+\nu}\right)^{2}\left(\frac{a}{b}\right)^{4}\left(\frac{1}{5}-\frac{18}{\left(\frac{a}{b}\right)^{-1} \pi^{5}}\right) \sum_{n=1}^{\infty}\left(\frac{\tanh \left(n \pi\left(\frac{a}{b}\right)^{-1}\right)}{n^{5}}\right)\right]^{-1}
\end{gathered}
$$




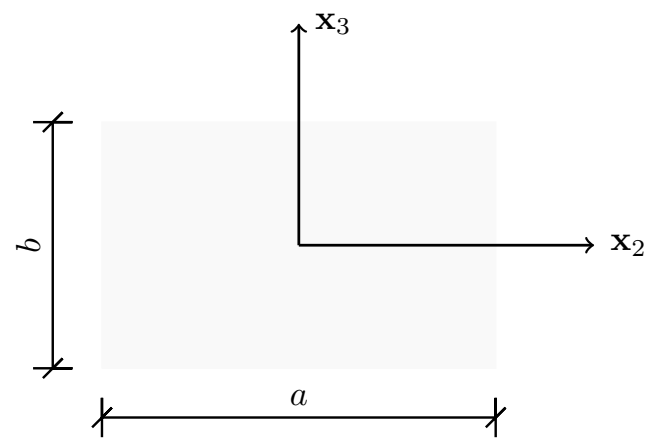

Figure 2: Rectangular beam cross-section dimensions

in which $a$ and $b$ are the beam rectangular cross-section dimensions (see Fig. 22).

Eqs. 2.19, 2.20, 2.22 and 2.23 in conjunction with the boundary conditions constitute a complete set of equations. The boundary conditions for the free vibration analysis of a non-rotating beam are represented in Table A.14.

\subsection{Linearization}

In order to cast the governing equations into an eigenvalue problem, a linearization about the steady state solution is performed at first. For this purpose the unknown variables are expressed as:

$$
\mathbf{\Psi}\left(x_{1}, t\right)=\mathbf{\Psi}_{0}\left(x_{1}\right)+\hat{\mathbf{\Psi}}\left(x_{1}, t\right)
$$

in which $\boldsymbol{\Psi}\left(x_{1}, t\right)$ is the state of the desired unknown variable, $\mathbf{\Psi}_{0}\left(x_{1}\right)$ is a steady state solution and $\widehat{\Psi}\left(x_{1}, t\right)$ is a small perturbation about the steady state position. The application of this linearization to Eqs. (2.19) and 2.20 will lead to the following equations:

$$
\begin{aligned}
& \widehat{\mathbf{F}}_{, 1}+\widetilde{\mathbf{K}}_{0} \cdot \widehat{\mathbf{F}}+\tilde{\widehat{\mathbf{K}}} \cdot \mathbf{F}_{0}+\overline{\mathbf{f}}=\dot{\hat{\mathbf{L}}}+\widetilde{\mathbf{\Omega}}_{0} \cdot \widehat{\mathbf{L}}+\tilde{\widehat{\mathbf{\Omega}}} \cdot \mathbf{L}_{0} \\
& \widehat{\mathbf{M}}_{1}+\widetilde{\mathbf{K}}_{0} \cdot \widehat{\mathbf{M}}+\tilde{\hat{\mathbf{K}}} \cdot \mathbf{M}_{0}+\left(\widetilde{\mathbf{e}}_{1}+\widetilde{\gamma}_{0}\right) \cdot \widehat{\mathbf{F}}+\widetilde{\widehat{\gamma}} \cdot \mathbf{F}_{0}+\overline{\mathbf{m}}=\dot{\hat{\mathbf{P}}}+\widetilde{\mathbf{\Omega}}_{0} \cdot \widehat{\mathbf{P}}+\widetilde{\hat{\mathbf{\Omega}}} \cdot \mathbf{P}_{0}+\tilde{\mathbf{V}}_{0} \cdot \widehat{\mathbf{L}}+\tilde{\hat{\mathbf{V}}} \cdot \mathbf{L}_{0} \\
& \widehat{\mathbf{V}}_{, 1}+\widetilde{\mathbf{K}}_{0} \cdot \hat{\mathbf{V}}+\widetilde{\hat{\mathbf{K}}} \cdot \mathbf{V}_{0}+\left(\widetilde{\mathbf{e}}_{1}+\widetilde{\gamma}_{0}\right) \cdot \hat{\boldsymbol{\Omega}}+\widetilde{\widehat{\gamma}} \cdot \boldsymbol{\Omega}_{0}=\dot{\hat{\gamma}} \\
& \widehat{\boldsymbol{\Omega}}_{, 1}+\widetilde{\mathbf{K}}_{0} \cdot \hat{\boldsymbol{\Omega}}+\tilde{\widehat{\mathbf{K}}} \cdot \boldsymbol{\Omega}_{0}=\dot{\hat{\boldsymbol{\kappa}}}
\end{aligned}
$$

where, $e_{1}=\left[\begin{array}{lll}1 & 0 & 0\end{array}\right]^{T}$. 


\subsection{Eigenvalue Problem}

For obtaining the eigenvalue problem, external forces and moments i.e. $\overline{\mathbf{f}}$ and $\overline{\mathbf{m}}$ are eliminated and unknown variables in Eq. (2.27) are discretized as:

$$
\begin{aligned}
\widehat{\mathbf{F}} & =\phi_{F}\left(x_{1}\right) \cdot \mathbf{q}_{F}(t) \\
\widehat{\mathbf{M}} & =\phi_{M}\left(x_{1}\right) \cdot \mathbf{q}_{M}(t) \\
\widehat{\mathbf{V}} & =\phi_{V}\left(x_{1}\right) \cdot \mathbf{q}_{V}(t) \\
\hat{\mathbf{\Omega}} & =\phi_{\Omega}\left(x_{1}\right) \cdot \mathbf{q}_{\Omega}(t)
\end{aligned}
$$

Based on the discretization given in Eq. 2.28 the free vibration equations can be written as:

$$
\mathcal{A} . \mathbf{q}=\mathcal{B} . \dot{\mathbf{q}}
$$

in which $\mathbf{q}=\left[\begin{array}{llll}\mathbf{q}_{F} & \mathbf{q}_{M} & \mathbf{q}_{V} & \mathbf{q}_{\Omega}\end{array}\right]^{T}$ and $\mathcal{A}$ and $\mathcal{B}$ are given as:

$$
\mathcal{A}=\left[\begin{array}{cccc}
\mathcal{A}_{11} & \mathcal{A}_{12} & \mathcal{A}_{13} & \mathcal{A}_{14} \\
\mathcal{A}_{21} & \mathcal{A}_{22} & \mathcal{A}_{23} & \mathcal{A}_{24} \\
\mathcal{A}_{31} & \mathcal{A}_{32} & \mathcal{A}_{33} & \mathcal{A}_{34} \\
\mathcal{A}_{41} & \mathcal{A}_{42} & {[0]} & \mathcal{A}_{44}
\end{array}\right], \quad \quad \mathcal{B}=\left[\begin{array}{cccc}
{[0]} & {[0]} & \mathcal{B}_{13} & \mathcal{B}_{14} \\
{[0]} & {[0]} & \mathcal{B}_{23} & \mathcal{B}_{24} \\
\mathcal{B}_{31} & \mathcal{B}_{32} & {[\mathbf{0}]} & {[\mathbf{0}]} \\
\mathcal{B}_{41} & \mathcal{B}_{42} & {[0]} & {[0]}
\end{array}\right]
$$

The entries of $\mathcal{A}$ and $\mathcal{B}$ are given in AppendixB.

\section{Chebyshev Collocation Discretization}

For the purpose of numerical discretization of the eigenvalue problem presented the Chebeyshev collocation method is utilized. In order to discretize the eigenvalue problem represented in Eq.2.29) Chebyshev polynomials (See AppendixC) are adopted as the spatial trial functions and Chebyshev points are employed as the collocation points. Chebyshev points are the roots of the Chebyshev polynomials of the first kind and in the interval $-1 \leqslant x \leqslant+1$ are:

$$
x_{i}=\cos \left(\frac{2 i-1}{2 N} \pi\right), \quad i=1,2, \ldots, N
$$

in which $N$ is the highest degree of the Chebyshev polynomials used as the trial functions. For an arbitrary interval $a \leqslant x \leqslant b$ the transformed Chebyshev points are:

$$
x_{i}=\frac{1}{2}(a+b)-\frac{1}{2}(b-a) \cos \left(\frac{2 i-1}{2 N} \pi\right), \quad i=1,2, \ldots, N
$$


Utilizing the Chebyshev polynomials as the trial functions in Eq. 2.29) and Eq. 2.30, we have:

$$
\phi_{F}\left(x_{1}\right)=\phi_{M}\left(x_{1}\right)=\phi_{V}\left(x_{1}\right)=\phi_{\Omega}\left(x_{1}\right)=\left[\begin{array}{ccc}
\psi\left(x_{1}\right) & {[0]} & {[0]} \\
{[0]} & \psi\left(x_{1}\right) & {[0]} \\
{[0]} & {[0]} & \psi\left(x_{1}\right)
\end{array}\right]
$$

where

$$
\psi\left(x_{1}\right)=\left[\begin{array}{lllll}
T_{0}\left(x_{1}\right) & T_{1}\left(x_{1}\right) & T_{2}\left(x_{1}\right) & \ldots & T_{N}\left(x_{1}\right)
\end{array}\right]
$$

Setting the residuals of Eq. 2.29 at $N$ Chebyshev points equal to zero in conjunction with 12 boundary conditions, constitute $12(N+1)$ equations, which can be expressed as:

$$
\text { A.X }=\text { B. } \dot{X}
$$

in which $\mathbf{A}$ and $\mathbf{B}$ are $12(N+1) \times 12(N+1)$ coefficient matrices with real numeric entries and $\mathbf{X}$ is the generalizied coordinates vector. $\mathbf{A}$ and $\mathbf{B}$ matrices have unsymmetrical structures and they are relatively sparse. If one assumes a harmonic solution; $\mathbf{X}=\overline{\mathbf{X}} e^{\lambda t}$, the generalized form of the eigenvalue problem is derived as:

$$
\mathbf{A} \cdot \overline{\mathbf{X}}=\mathbf{B} \cdot \lambda \overline{\mathbf{X}}
$$

where $\lambda$ is the eigenvalue or the natural frequency of the problem.

\section{Numerical Results}

In order to show the applicability, validity and accuracy of the proposed collocation scheme, illustrative examples are presented in this section and the obtained results are compared to the analytical, experimental as well as other numerical schemes.

\subsection{Numerical Results for Straight Beams}

Comparison to Galerkin's and FEM Methods. Traugott et al. (2006) and Patil and Althoff (2011) based on Galerkin's and finite element method discretization of the intrinsic formulation of geometrically exact beams have considered a cantilever beam and have presented numerical results for natural frequencies. The structural and inertial properties of the cantilever beam are presented in Table(1). In order to show the convergence of the Chebyshev collocation method the first few natural frequencies of the cantilever beam are presented in Table $(2)$ in which $N$ is the highest degree of Chebyshev polynomials used in the analysis. 
Table 1: Cantilever Beam Properties

\begin{tabular}{ll}
\hline Span & $16 \mathrm{~m}$ \\
Chord & $1 \mathrm{~m}$ \\
Mass per Unit Length & $0.75 \mathrm{~kg} / \mathrm{m}$ \\
Mass Moment of Inertia per Unit Length & $0.1 \mathrm{~kg} . \mathrm{m}$ \\
Spanwise Elastic Axis & $50 \%$ Chord \\
Center of Gravity & $50 \%$ Chord \\
Flapwise Bending Rigidity & $2 \times 10^{4} \mathrm{~N} . \mathrm{m}^{2}$ \\
Torsional Rigidity & $1 \times 10^{4} \mathrm{~N} . \mathrm{m}^{2}$ \\
Chordwise Bending Rigidity & $4 \times 10^{6} \mathrm{~N} . \mathrm{m}^{2}$ \\
Shear/Extensional Rigidity & $\infty$ \\
\hline
\end{tabular}

As it is seen a fast rate of convergence is observed at fairly low degree of polynomials.

In Table 3 the natural frequencies obtained based on the current approach are compared with those of Traugott et al. (2006) that is based on a finite element (FEM) scheme and Patil and Althoff (2011) which is based on a Galerkin's method, as well as the exact solution. As it is seen a very good accuracy is exhibited by the current approach compared to the exact frequencies. The accuracy of the results based on the current approach is obviously superior to those of Traugott et al. (2006) but Patil and Althoff (2011) have predicted the natural frequencies of the bending modes more accurately compared to the current approach. One may seek the reason in the fact that the collocation methods generally lead to unsymmetric matrices while Galerkin's methods lead to symmetric ones.

Yeo et al. (2014) have considered a comparison of free vibration analysis based on 3D finite element and $1 \mathrm{D}$ beam elements. The 3D finite element analysis has been performed using a commercial code MSC/Marc and the 1D beam analysis is performed using rotorcraft comprehensive analysis code RCAS. Two versions of nonlinear beam element namely NLB and GCB have been used in the analysis. RCAS NLB beam element is based on a moderately large deflection beam theory and RCAS GCB is based on a geometrically exact beam theory. An aluminum beam with three different aspect ratios with length of $L=20 \times c, L=10 \times c$ and $L=5 \times c$ has been considered. The beam properties used in the analysis are as follows; $E=1.0 \times 10^{7} \frac{l b}{i n^{2}}, \quad \nu=0.3, \quad \rho=0.098 \frac{l b}{i n^{3}}, \quad c=3.4 \mathrm{in}, \quad t=\frac{c}{4}$ where $c$ and $t$ are the beam cross-sectional chord and thickness. Tables (4) and (5) show the first torsion frequency and the third flapwise frequency, respectively. As it is seen the results based on the current approach are in a very good agreement with 3D as well as 1D finite elements. 
Table 2: Convergence of the Chebyshev Collocation Method

\begin{tabular}{lcccc}
\hline & \multicolumn{4}{c}{$N$ (Highest Degree of Polynomials) } \\
\cline { 2 - 5 } & 4 & 6 & 8 & 10 \\
\hline 1st Bending & 2.241 & 2.242 & 2.242 & 2.242 \\
2nd Bending & 14.71 & 14.00 & 14.00 & 14.00 \\
3rd Bending & 65.32 & 39.46 & 38.97 & 38.96 \\
1st Torsion & 31.69 & 31.05 & 31.05 & 31.05 \\
2nd Torsion & 95.25 & 93.13 & 93.14 & 93.14 \\
\hline
\end{tabular}

Comparison to Experimental Results. Dowell and Traybar (1975) have conducted a series of experiments for large deflections and natural frequencies of two cantilever beams. The straight aluminium ( $\mathrm{T}$ 7075) beams are of $20 \mathrm{in}$ and $30 \mathrm{in}$ length respectively with uniform rectangular cross-sections of $0.5 \mathrm{in} \times 0.125 \mathrm{in}$. The properties of these two beams are listed as follows;

$E=10.576 \times 10^{6} \frac{\mathrm{lb}}{i n^{2}}, \quad G=4.0383 \times 10^{6} \frac{\mathrm{lb}}{i n^{2}}, \quad \nu=0.31, \quad \rho=0.1014 \frac{\mathrm{lb}}{i n^{3}}$

Table (6) shows the 1st flapwise and 1st chordwise natural frequencies based on the current approach and the experimental results of Dowell and Traybar (1975). It is seen a very good agreement between experimental and numerical results. It is noted that the experimental results given in Table (6) are averaged of a series of test values represented by Dowell and Traybar (1975).

Results for Various Boundary Conditions. Free vibration of beams with various boundary conditions common in the engineering and science problems introduced in Section 2.2 (see Table (A.14)) are considered herein. The beam properties used here are those from Table (1). Tables 77 through 10 show the obtained 10 first natural frequencies using present approach and they are compared to the exact analytical results (Meirovitch (1997)). A very good correlation is observed for all boundary conditions.

Large Deflected Cantilever. The nonlinear free vibration behavior of a cantilever beam deflected under a follower end load is considered. This problem has been introduced by Patil et al. (1999) and also considered by Patil and Althoff (2011). Under the action of the tip load the beam will undergo large deflections. The large deflected position is considered as the steady state position of the beam and the natural frequencies are obtained for various levels of the tip load. It is noted that the calculations of the steady state (here static) position of a cantilever beam under static loads using Chebyshev collocation method are thoroughly considered in Khaneh Masjedi and Ovesy (2015). Large deflections of the beam cause a coupling between chordwise bending and torsion modes. Fig. (3) depicts the first few natural frequencies of the large deflected 


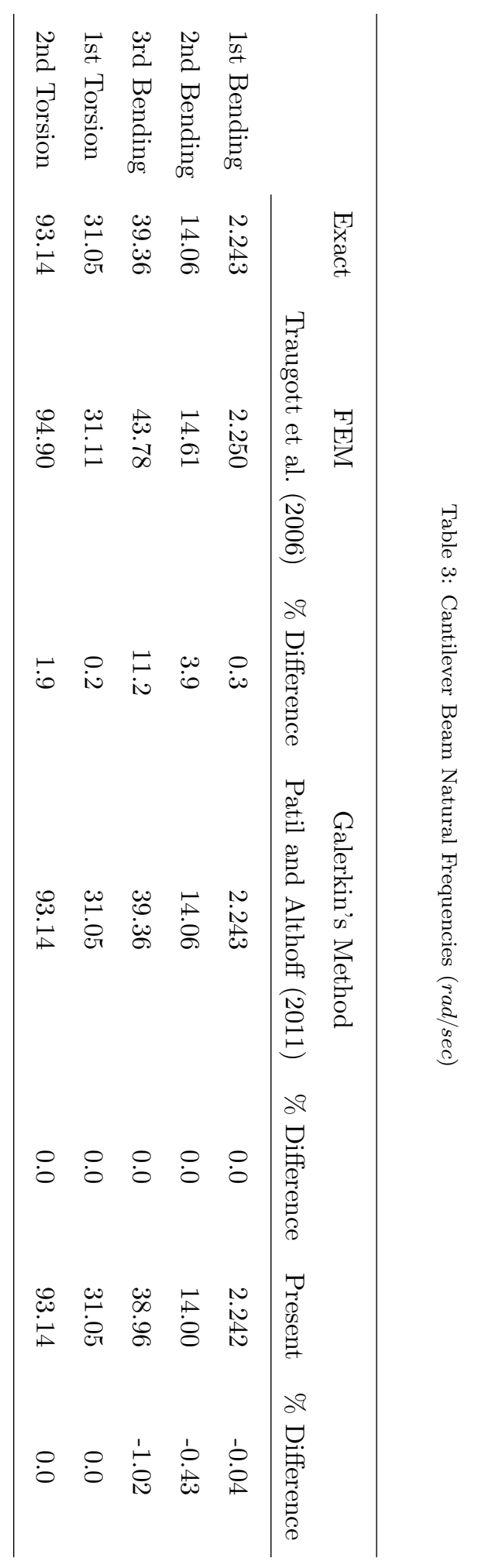


Table 4: 1st Torsion Frequency of Aluminum Beam $(H z)$

\begin{tabular}{|c|c|c|c|c|c|}
\hline & & & $L=20 \times c$ & $L=10 \times c$ & $L=5 \times c$ \\
\hline Yeo et al. & $(2014)$ & RCAS NLB & 201.54 & 403.08 & 806.17 \\
\hline Yeo et al. & $(2014)$ & RCAS GCB & 201.54 & 403.08 & 806.17 \\
\hline Yeo et al. & $(2014)$ & MSC/Marc & 201.41 & 412.93 & 842.36 \\
\hline Present & & & 201.45 & 402.89 & 805.79 \\
\hline
\end{tabular}

Table 5: 3rd Flapwise Frequency of Aluminum Beam $(H z)$

\begin{tabular}{|c|c|c|c|c|c|}
\hline & & & $L=20 \times c$ & $L=10 \times c$ & $L=5 \times c$ \\
\hline Yeo et al. & (2014) & RCAS NLB & 103.36 & 412.94 & 1641.87 \\
\hline Yeo et al. & (2014) & RCAS GCB & 103.20 & 410.28 & 1603.10 \\
\hline Yeo et al. & (2014) & MSC/Marc & 104.00 & 413.94 & 1623.61 \\
\hline Present & & & 103.29 & 411.59 & 1621.88 \\
\hline
\end{tabular}

Table 6: Princeton Beam Natural Frequencies $(H z)$

\begin{tabular}{|c|c|c|c|}
\hline \multirow[b]{2}{*}{$L=20 \mathrm{in}$} & \multirow[t]{2}{*}{ Experiment } & \multirow[t]{2}{*}{ Present } & \multirow[t]{2}{*}{$\%$ Difference } \\
\hline & & & \\
\hline Flapwise Bending & 10.150 & 10.153 & -0.030 \\
\hline Chordwise Bending & 41.143 & 40.553 & 1.434 \\
\hline \multicolumn{4}{|l|}{$L=30 \mathrm{in}$} \\
\hline Flapwise Bending & 4.509 & 4.406 & -2.284 \\
\hline Chordwise Bending & 17.207 & 18.001 & 4.614 \\
\hline
\end{tabular}


Table 7: First 10 Natural Frequencies for C-C Boundary Conditions $(\mathrm{rad} / \mathrm{sec})$

\begin{tabular}{llcc}
\hline$\#$ & Mode & Exact & Present \\
\hline 1 & 1st Flapwise Bending & 14.27 & 14.25 \\
2 & 2nd Flapwise Bending & 39.34 & 39.11 \\
3 & 1st Torsion & 62.09 & 62.09 \\
4 & 3rd Flapwise Bending & 77.12 & 76.16 \\
5 & 1st Chordwise Bending & 100.91 & 100.75 \\
6 & 2nd Torsion & 124.18 & 124.18 \\
7 & 4th Flapwise Bending & 127.49 & 125.28 \\
8 & 3rd Torsion & 186.27 & 186.27 \\
9 & 5th Flapwise Bending & 190.44 & 191.82 \\
10 & 4th Torsion & 248.36 & 248.5 \\
\hline
\end{tabular}

Table 8: First 10 Natural Frequencies for S-S Boundary Conditions $(\mathrm{rad} / \mathrm{sec})$

\begin{tabular}{llcc}
\hline$\#$ & Mode & Exact & Present \\
\hline 1 & 1st Flapwise Bending & 6.29 & 6.29 \\
2 & 2nd Flapwise Bending & 25.18 & 25.05 \\
3 & 1st Chordwise Bending & 44.52 & 44.46 \\
4 & 3rd Flapwise Bending & 56.66 & 56.02 \\
5 & 1st Torsion & 62.09 & 62.09 \\
6 & 4th Flapwise Bending & 100.73 & 98.83 \\
7 & 2nd Torsion & 124.18 & 124.18 \\
8 & 5th Flapwise Bending & 157.39 & 154.75 \\
9 & 2nd Chordwise Bending & 178.07 & 177.16 \\
10 & 3rd Torsion & 186.27 & 186.27 \\
\hline
\end{tabular}


Table 9: First 10 Natural Frequencies for S-C Boundary Conditions $(\mathrm{rad} / \mathrm{sec})$

\begin{tabular}{llcc}
\hline$\#$ & Mode & Exact & Present \\
\hline 1 & 1st Flapwise Bending & 9.83 & 9.82 \\
2 & 1st Torsion & 31.04 & 31.04 \\
3 & 2nd Flapwise Bending & 31.87 & 31.69 \\
4 & 3rd Flapwise Bending & 66.50 & 65.70 \\
5 & 1st Chordwise Bending & 69.54 & 69.44 \\
6 & 2nd Torsion & 93.13 & 93.13 \\
7 & 4th Flapwise Bending & 113.72 & 111.60 \\
8 & 3rd Torsion & 155.22 & 155.23 \\
9 & 5th Flapwise Bending & 173.52 & 172.19 \\
10 & 4th Torsion & 217.31 & 217.34 \\
\hline
\end{tabular}

Table 10: First 10 Natural Frequencies (Excluding Rigid Body Mode) for F-F Boundary Conditions ( rad/sec)

\begin{tabular}{llcc}
\hline$\#$ & Mode & Exact & Present \\
\hline 1 & 1st Flapwise Bending & 14.87 & 14.18 \\
2 & 2nd Flapwise Bending & 39.34 & 38.79 \\
3 & 1st Torsion & 62.09 & 62.09 \\
4 & 3rd Flapwise Bending & 77.12 & 75.32 \\
5 & 1st Chordwise Bending & 100.91 & 100.27 \\
6 & 4th Flapwise Bending & 127.49 & 123.5 \\
7 & 2nd Torsion & 124.18 & 124.18 \\
8 & 3rd Torsion & 186.27 & 186.27 \\
9 & 5th Flapwise Bending & 190.44 & 187.88 \\
10 & 4th Torsion & 248.36 & 248.50 \\
\hline
\end{tabular}


Table 11: Coupled Flap/Chord-Wise Natural Frequencies $(H z)$ for $45^{\circ}$ Pretwisted Beam

\begin{tabular}{|c|c|c|c|}
\hline Mode \# & Experiment Carnegie (1959) & Present & $\%$ Difference \\
\hline 1 & 59.0 & 62.1 & 5.25 \\
\hline 2 & 290.0 & 305.3 & 5.28 \\
\hline 3 & 920.0 & 943.8 & 2.59 \\
\hline 4 & 1110.0 & 1200.0 & 8.12 \\
\hline
\end{tabular}

beam. The beam properties used in the analysis are given in Table(1). As it is seen while the flapwise bending modes are not as much sensitive to the tip deflections, coupled torsion-chordwise bending modes are quite dependent on the beam tip deflections. It is noted that the current behavior is in a very good agreement with those presented by Patil et al. (1999) or Patil and Althoff (2011).

\subsection{Numerical Results for Pre-Twisted Beams}

Comparison to Experimental Results. Coupled flap/chord-wise bending vibration of a pretwisted Timoshenko beam with a total twist of $45^{\circ}$ has been experimentally considered by Carnegie (1959). The problem is devised in such a way to decouple torsional vibration from flap/chord-wise bending vibration by choosing a very high magnitude of torsional stiffness (i.e. $G J=\infty$ ). The properties of this pretwisted cantilever is listed as follows;

$L=0.1524 m, \quad E I_{2}=2.26 N . m^{2}, \quad E I_{3}=487.9 N . m^{2}, \quad G A_{2}=G A_{3}=3.076 \times 10^{6}, \quad \mu=0.3447 \mathrm{~kg} / \mathrm{m}$. Table (11) depicts a comparison of current approach with experimental results of Carnegie (1959) for the first four natural coupled bending frequencies. A it is observed, there is a very good agreement between two sets of results.

Non-Rotating Blade. A twisted cantilever blade sample, introduced by Rosen et al. (1987) is considered. The blade has a zero twist at root and $40^{\circ}$ twist at tip, other properties are as follows;

$L=3.048 m, \quad E=70 \times 10^{9} \mathrm{~N} / \mathrm{m}, \quad G=27 \times 10^{9} \mathrm{~N} / \mathrm{m}, \quad \rho=2700 \mathrm{~kg} / \mathrm{m}^{3}, \quad A=0.0127667 \mathrm{~m}^{2}$, $E I_{2}=2869.7 N . m^{2}, \quad E I_{3}=57393 N . m^{2}, \quad c_{2}=\frac{2}{3}, \quad c_{3}=\frac{5}{8}, \quad \mu=34.47 \mathrm{~kg} / \mathrm{m}$.

A comparison of Euler-Bernoulli theory and Timoshenko theory is carried out in Table 12 and also compared to the numerical results of Banerjee (2004) which are obtained by the method of exact dynamic stiffness matrix. The results based on the two numerical sets are in a very good concordance. For the present case a very slight difference is seen between Timoshenko and Euler-Bernoulli theories and as expected Timoshenko theory has predicted the natural frequencies slightly lower compared to the Euler-Bernoulli theory. This negligible difference between two theories is attributed to the fact that the cross-sectional dimensions 


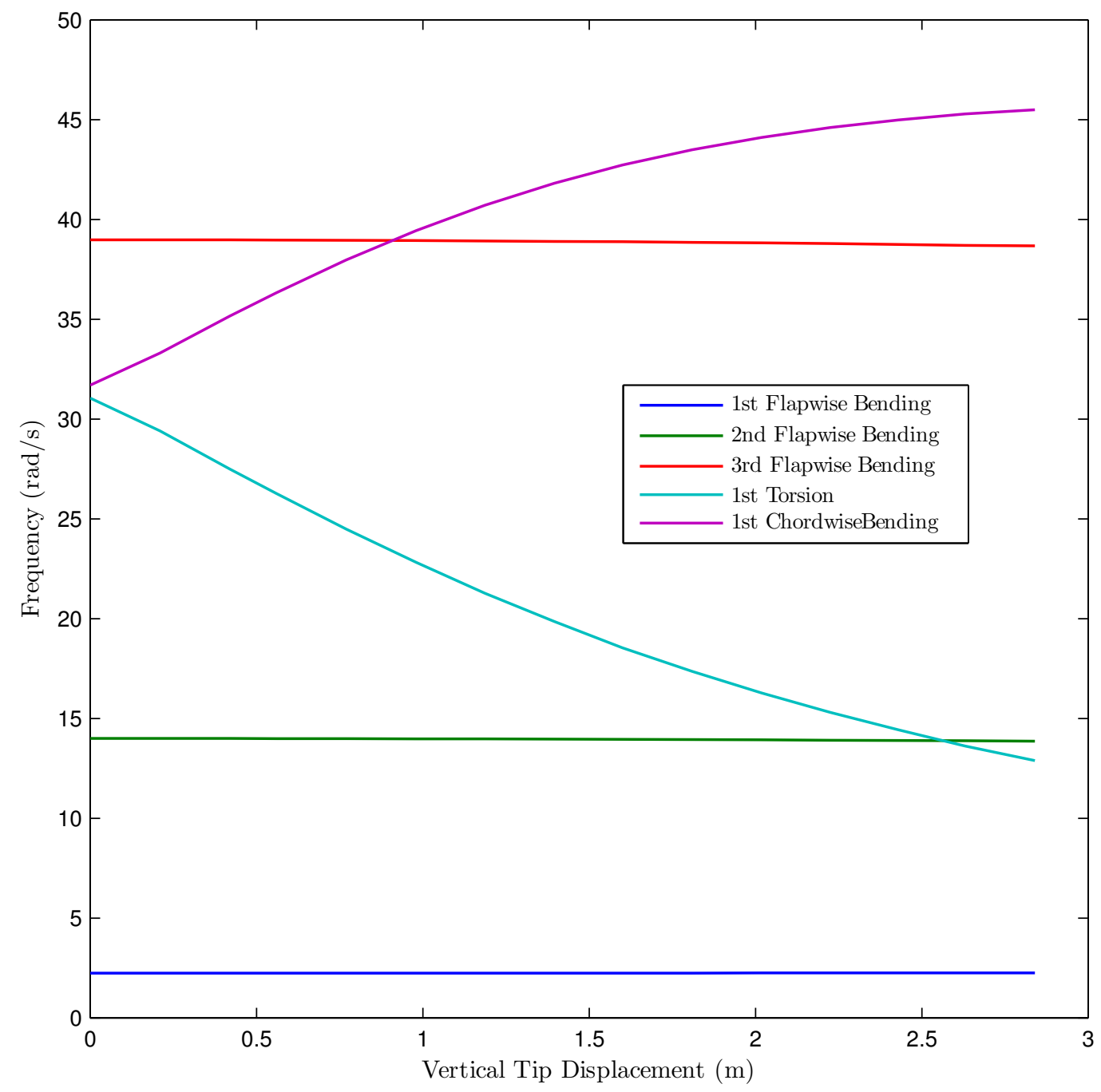

Figure 3: Variation of Natural Frequencies with Tip Deflection 
Table 12: Non-Rotating Twisted Blade Natural Frequencies ( $\mathrm{rad} / \mathrm{sec}$ )

\begin{tabular}{|c|c|c|c|c|}
\hline \multirow[b]{2}{*}{ Mode \# } & \multicolumn{2}{|c|}{ Euler-Bernoulli Theory } & \multicolumn{2}{|c|}{ Timoshenko Theory } \\
\hline & Banerjee (2004) & Present & Banerjee (2004) & Present \\
\hline 1 & 3.4717 & 3.4719 & 3.4715 & 3.4718 \\
\hline 2 & 13.3465 & 13.3413 & 13.340 & 13.3408 \\
\hline 3 & 25.1707 & 25.1666 & 25.615 & 25.1653 \\
\hline 4 & 56.3716 & 56.3673 & 56.363 & 56.3634 \\
\hline 5 & 103.263 & 103.2529 & 103.20 & 103.2111 \\
\hline
\end{tabular}

of this particular beam is considerably lower compared to the beam span which makes this sample blade extremely slender and as a result the shear deformation does not have a significant impact on the natural frequencies.

The Effects of Pretwist Angle and Shear Deformation. In order to show the effects of the overall twist and shear deformations on the natural frequencies of pretwisted beams, the following example is introduced. A cantilever beam with zero twist at root and a range of tip twist from zero to $180^{\circ}$ is considered. The natural frequencies are obtained based on either Timoshenko or Euler-Bernoulli theories. The properties used for this example are as follows;

$L=1 \mathrm{~m}, \quad E=9 \times 10^{10} \mathrm{~N} / \mathrm{m}, \quad G=3.46 \times 10^{10} \mathrm{~N} / \mathrm{m}, \quad \rho=7850 \mathrm{~kg} / \mathrm{m}^{3}, \quad a=0.2 \mathrm{~m}, \quad b=0.1 \mathrm{~m}$.

Based on the Eqs. 2.25) the shear correction values are obtained as; $c_{2}=0.832942$ and $c_{3}=0.784442$. The first seven natural frequencies are depicted in Fig.(4). For the case of torsional modes the values for both theories are very close while for bending dominant modes (either flapwise or chordwise) the predicted results based on Timoshenko theory are lower compared to the Euler-Bernoulli theory and the difference between two theories becomes more remarkable by increasing the frequency of the bending dominant modes. The natural frequencies for both theories, qualitatively follow very similar trends with the increase of pretwist. The 1st flapwise and chordwise bending frequencies as well as either 1st or 2nd torsional frequencies remains relatively constant with the increase of the pretwist while the 2nd flapwise bending frequency is increasing and the 2nd chordwise bending frequency is decreasing. The 3rd flapwise bending has an increasing trend up to around $150^{\circ}$ tip twist and then it starts to decrease slightly.

NREL 5 MW Reference Wind Turbine Rotor Blade. In order to show the applicability and versatility of current approach the free vibration problem of a $5 \mathrm{MW}$ baseline wind turbine rotor blade, introduced by 


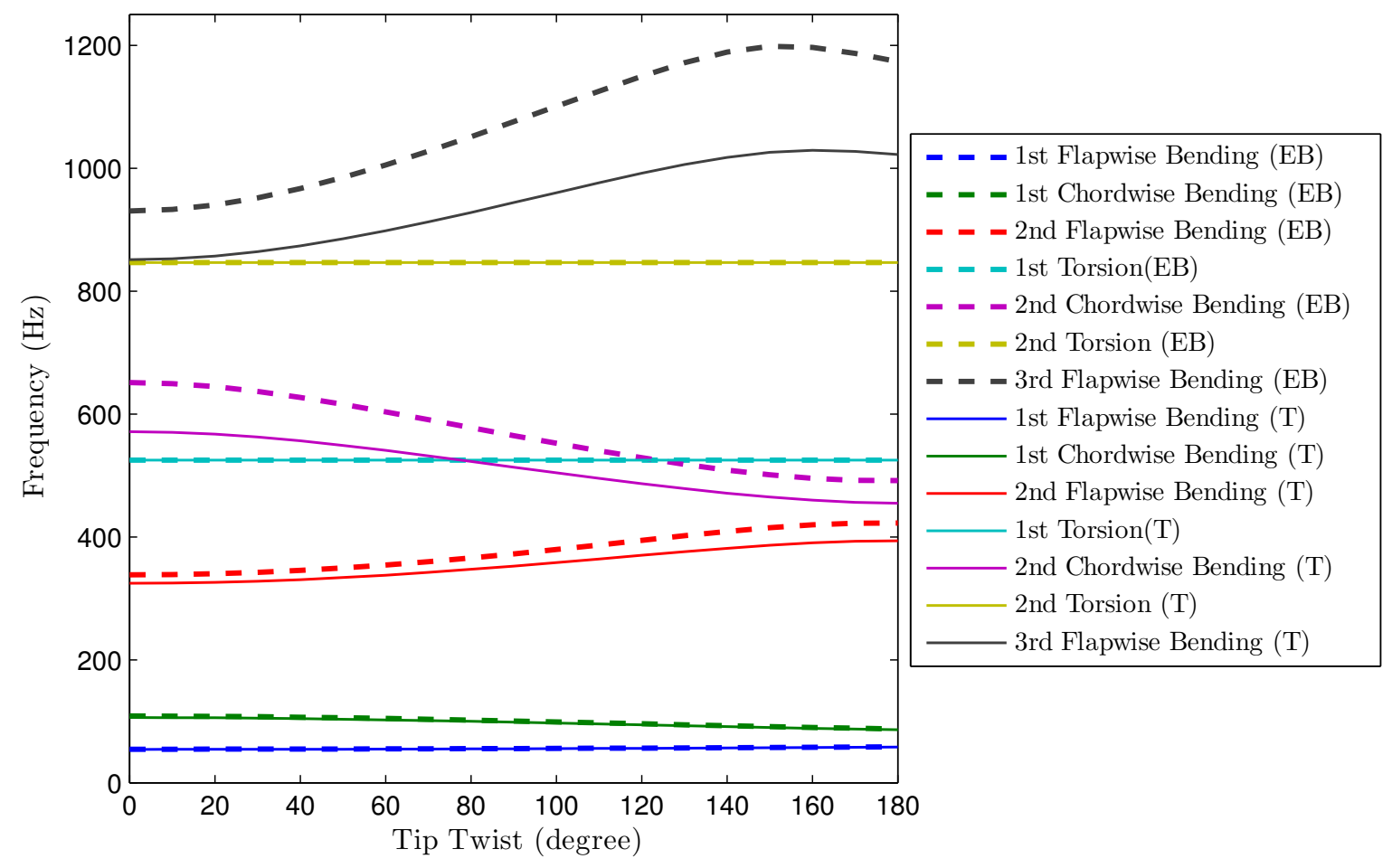

Figure 4: Variation of the First Seven Natural Frequencies of a Cantilever Beam with Tip Twist (EB: Euler-Bernoulli Theory, $\mathrm{T}$ : Timoshenko Theory) 


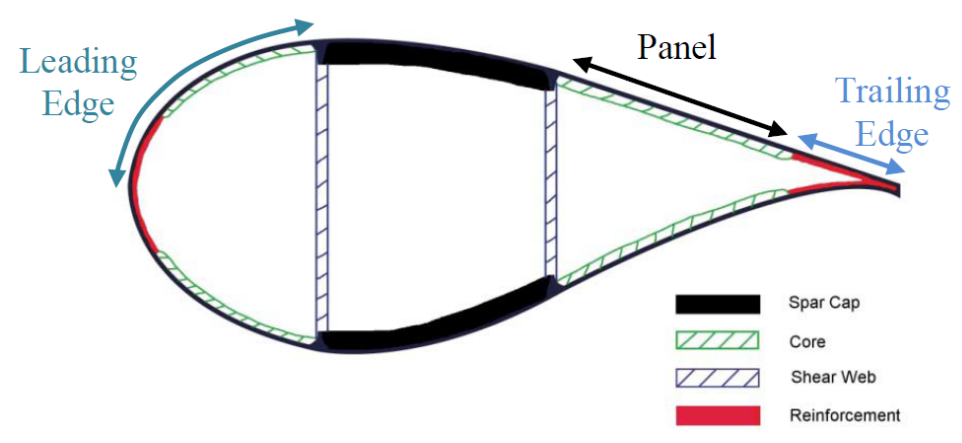

Figure 5: Representative Airfoil Cross Section with Two Shear Webs (from Griffith and Ashwill (2011))

Jonkman et al. (2009) is studied. A basic structural concept of the design of NREL 5 MW blades is presented by Resor (2013) (See Fig. (5)). The rotor blade has a non-uniform distribution of twist, mass, inertial and stiffness properties along its span. Figs. (6) through (8) show rotor blade twist, mass and stiffness properties respectively. These spanwise distributed properties which are obtained from Jonkman et al. (2009) are used as the input for the beam cross-sectional stiffness $\left(E A, G J, E I_{3}\right.$ and $\left.E I_{2}\right)$ and inertial properties as well as its twist value needed in every collocation points. Since no shear stiffness properties are reported in Jonkman et al. (2009), an Euler-Bernoulli theory is used in this sample.

The first lowest 6 modes are calculated for the case of non-rotating (i.e. $\bar{\Omega}=0 \mathrm{rpm}$ ) as well as rotating (i.e. $\bar{\Omega}=12.1 \mathrm{rpm}$ ) blade. It should be noted that the boundary conditions for a C-F rotating beam are presented as; $\left.\mathbf{V}\right|_{x_{1}=0}=0 ;\left.\quad \boldsymbol{\Omega}\right|_{x_{1}=0}=\overline{\boldsymbol{\Omega}}_{0} ;\left.\quad \mathbf{F}\right|_{x_{1}=L}=0 ;\left.\quad \mathbf{M}\right|_{x_{1}=L}=0$. Current results which are obtained using Chebyshev polynomials of maximum degree of 14 are compared in Table 13 to those presented by Jeong et al. (2014) based on simulation with BModes, FAST and their in-house FEM code with 17 four-noded cubic elements. A very good agreement is observed between current approach and other numerical results. The beauty of present paradigm lies in the fact that by using continuous polynomials with a relatively low degree, results with comparable accuracy as distinct from those of an FEM is obtained for a real life engineering sample problem with non-uniform properties. Campbell diagram which shows the variation of the first 6 modes with rotor blade rotational speed is depicted for this problem in Fig. (9) for a range of rotational speed; $\bar{\Omega}=0 \sim 15 \mathrm{rad} / \mathrm{s}$.

\section{Conclusions}

A Chebyshev collocation method for the eigenvalue analysis of the fully intrinsic equations of geometrically exact beams has been presented. The intrinsic formulation which is free from any rotational or displacement variables is desirable for numerical analysis due to its low order of nonlinearity which is quadratic at most. The Chebyshev collocation method which is based on the Chebyshev points as the collocation 


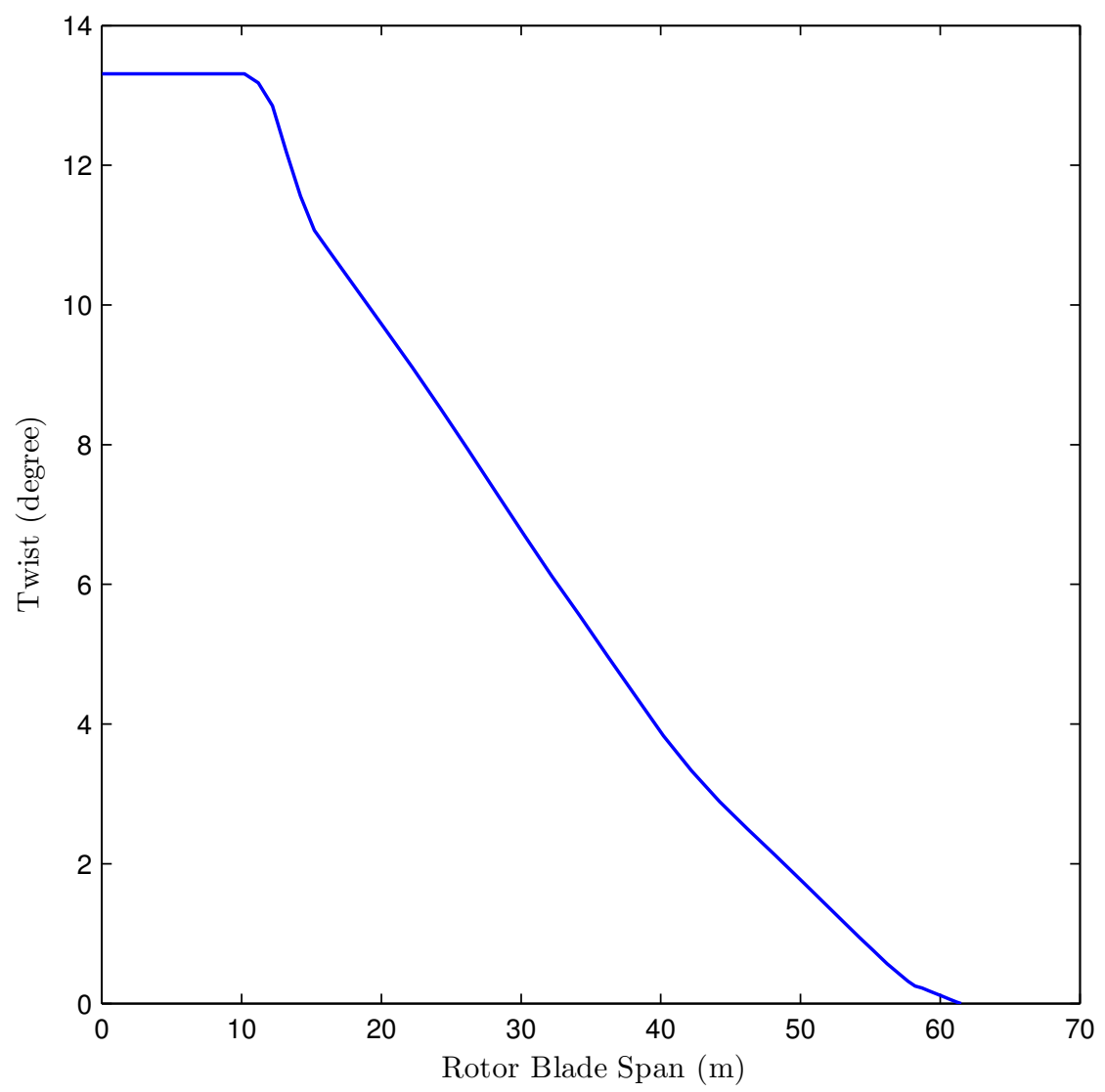

Figure 6: Twist Distribution of the NREL 5 MW reference wind turbine blade 


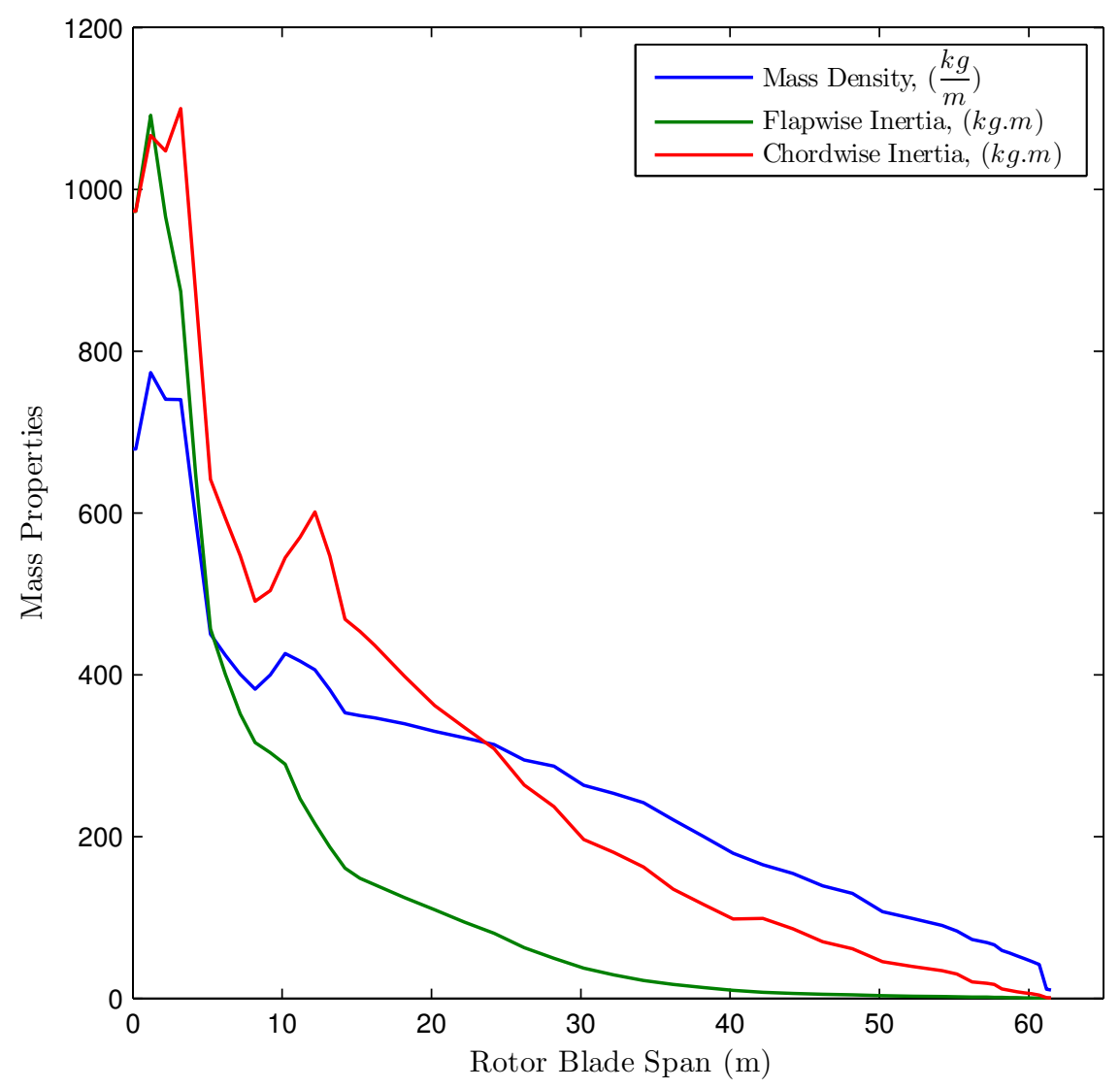

Figure 7: Mass and Inertial Properties of the NREL 5 MW reference wind turbine blade

Table 13: NREL 5 MW reference wind turbine blade natural frequencies $(H z)$

\begin{tabular}{|c|c|c|c|c|c|c|c|c|}
\hline \multirow[b]{2}{*}{ Mode \# } & \multicolumn{4}{|c|}{$\bar{\Omega}=0 \mathrm{rpm}$} & \multicolumn{4}{|c|}{$\bar{\Omega}=12.1 \mathrm{rpm}$} \\
\hline & BModes* & FAST* & Jeong et al. (2014) & Present & BModes* & FAST* & Jeong et al. (2014) & Present \\
\hline 1 & 0.68 & 0.69 & 0.694 & 0.692 & 0.73 & 0.74 & 0.744 & 0.741 \\
\hline 2 & 1.10 & 1.12 & 1.085 & 1.098 & 1.11 & 1.12 & 1.095 & 1.104 \\
\hline 3 & 1.94 & 2.00 & 1.997 & 2.001 & 2.00 & 2.06 & 2.036 & 2.057 \\
\hline 4 & 4.00 & 4.12 & 4.019 & 4.099 & 4.02 & 4.14 & 4.036 & 4.117 \\
\hline 5 & 4.43 & 4.64 & 4.479 & 4.672 & 4.48 & 4.69 & 4.535 & 4.723 \\
\hline 6 & 5.77 & 5.61 & 5.610 & 5.609 & 5.78 & 5.61 & 5.613 & 5.611 \\
\hline
\end{tabular}

* Taken from Jeong et al. (2014) 


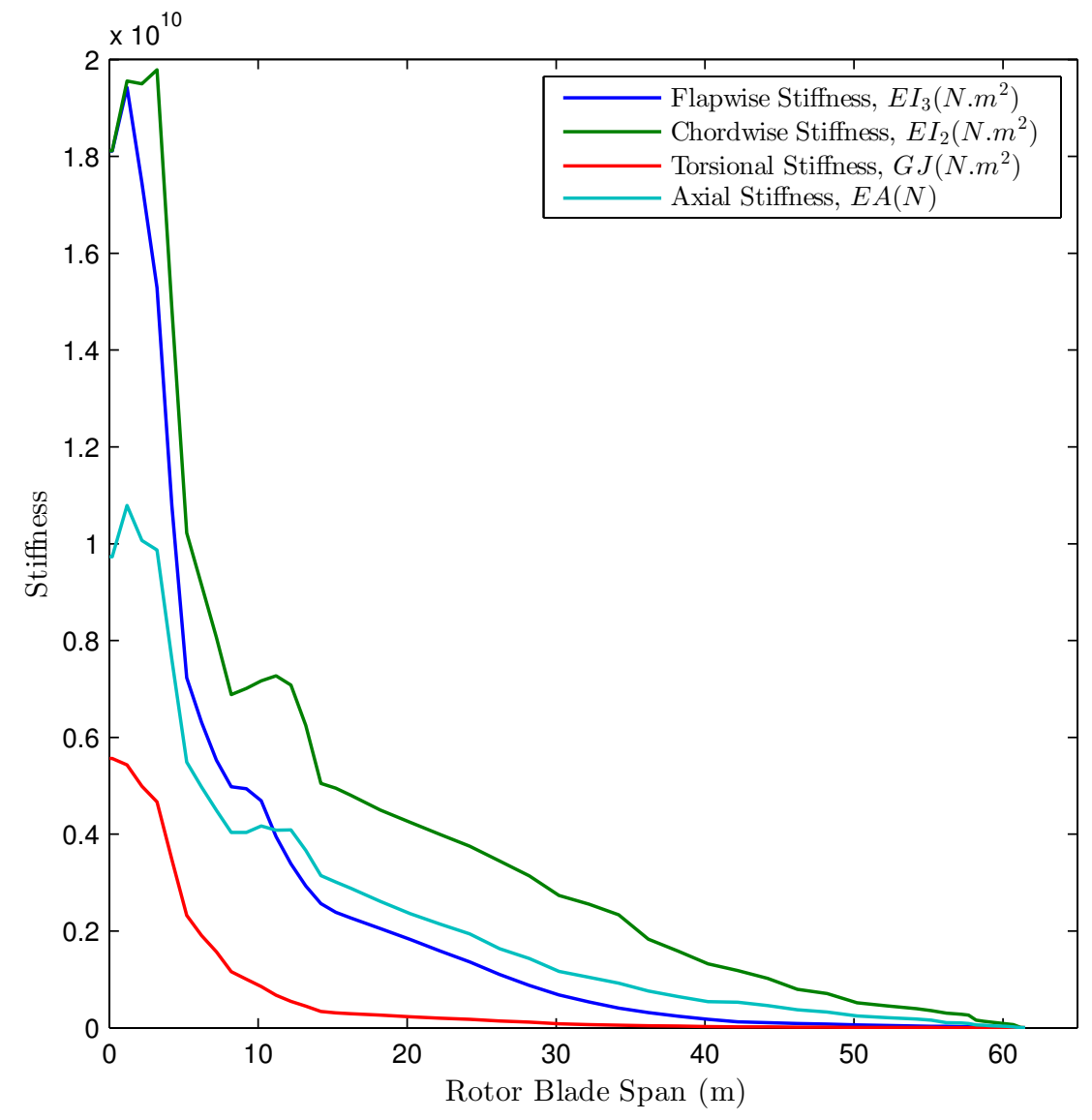

Figure 8: Stiffness Properties of the NREL 5 MW reference wind turbine blade 


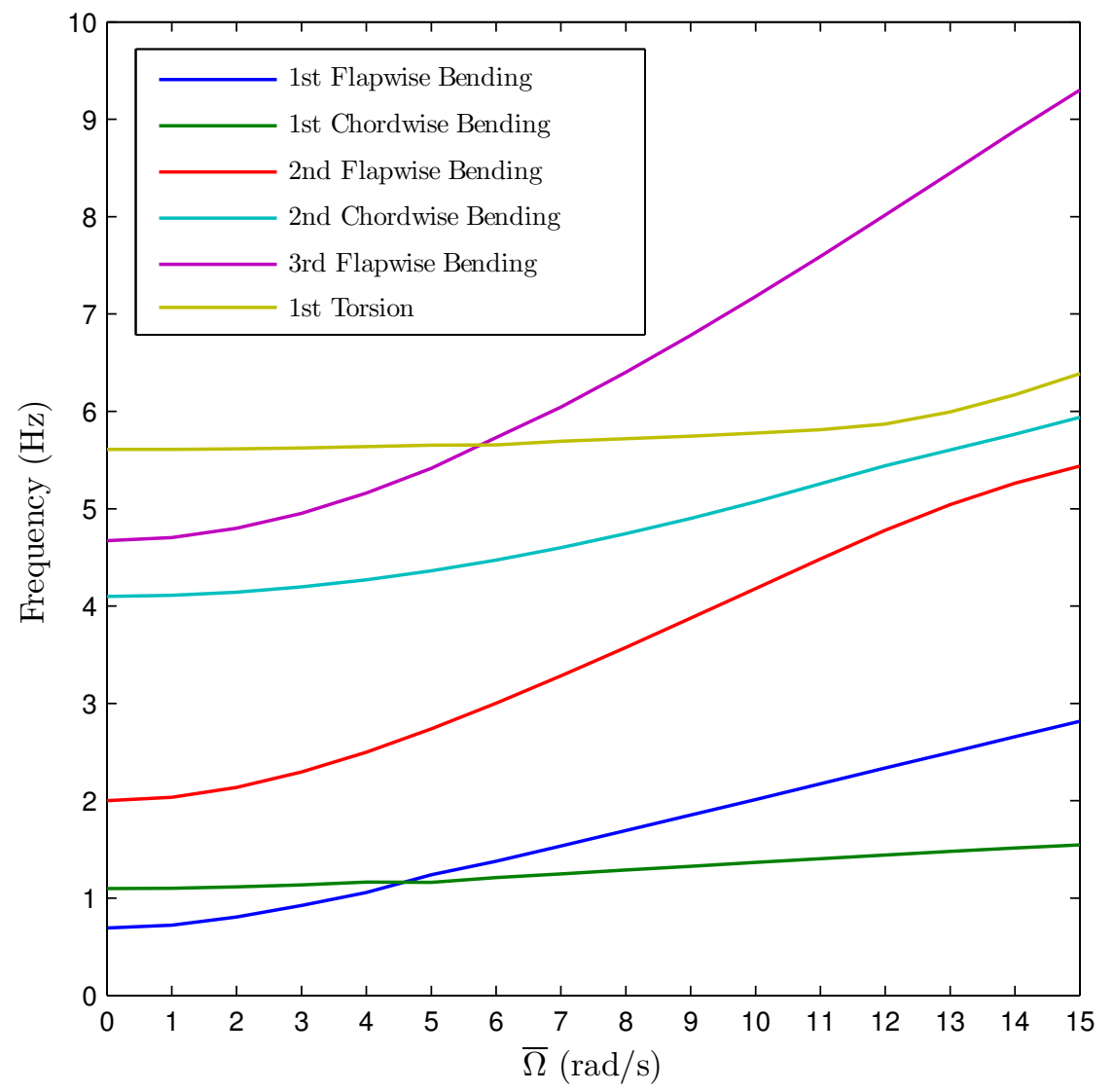

Figure 9: Campbell Diagram of the NREL 5 MW reference wind turbine blade 
points and the Chebyshev polynomials as the trial functions, contains no integration which is common in the Galerkin's and finite element methods. This make the Chebyshev collocation method computationally efficient in comparison to the finite element-based methods. It is very simple to implement and exhibits a very good rate of convergence in a relatively low order of polynomials. The accuracy and applicability of the proposed method for the free vibration analysis is shown for a number of straight and pretwisted beam samples and the obtained results are compared to the analytical, experimental as well as other numerical results. The versatility of the current approach has been shown by successfully tackling a real-life engineering problem of a composite wind turbine rotor blade with non-uniformly distributed properties. The capability of current approach to capture complicated engineering problems with comparable accuracy as distinct from FEM, introduces a promising paradigm having the advantages such as; simplicity, good accuracy and computational efficiency. All these desiring characteristics are obtained by merely employing continuous functions of polynomial type with fairly low order which even adds more to the beauty and merits of the presented approach. In summary, the characteristics that support the versatility of current approach are as follows:

1) There are no simplifying assumptions in the kinematical description (hence the name Geometrically Exact) and as a result 3D arbitrarily large deflections can be captured by this model.

3) Initial twist and curvatures are easily incorporated in this model without the need for any further theoretical developments.

4) The beam model is not restricted by constitutive equations and it is applicable to beam-like structures made from either isotropic or anisotropic materials with arbitrary cross-sectional shapes.

5) It is suitable for applications with non-uniform distribution of properties.

6) It can be further extended for hybrid schemes such as Strong Formulation Finite Element Method for more complex situations which require several beam assemblage.

Based on the above characteristics, current model can be directly applied in many real life engineering problems including aircraft wings, helicopter rotor blades, wind turbine rotor blades, space booms, moored and towed cables, etc. Additionally, the potential of current model in adopting hybrid numerical schemes such as Strong Formulation Finite Element Method makes it suitable for tackling more complicated problems in which several beam assemblage are required.

\section{References}

Banerjee, J.R., 2004. Development of an exact dynamic stiffness matrix for free vibration analysis of a twisted timoshenko beam. Journal of Sound and Vibration 270, 379-401.

Cardona, A., Géradin, M., 1988. A beam finite element non-linear theory with finite rotations. International Journal for Numerical Methods in Engineering 26, 2403-2438.

Carnegie, W., 1959. Vibrations of pre-twisted cantilever blading. Proceedings of the Institution of Mechanical Engineers 173, $343-374$. 
Chang, C.S., Hodges, D.H., 2009a. Stability studies for curved beams. Journal of Mechanics of Materials and Structures 4, $1257-1270$.

Chang, C.S., Hodges, D.H., 2009b. Vibration characteristics of curved beams. Journal of Mechanics of Materials and Structures 4, 675-692.

Dowell, E.H., Traybar, J.J., 1975. An experimental study of the nonlinear stiffness of a rotor blade undergoing flap, lag and twist deformations. AMS Report No. 1194 .

Genoese, A., Genoese, A., Bilotta, A., Garcea, G., 2014. A generalized model for heterogeneous and anisotropic beams including section distortions. Thin-Walled Structures 74, 85-103.

Ghorashi, M., Nitzsche, F., 2009. Nonlinear dynamic response of an accelerating composite rotor blade using perturbations. Journal of Mechanics of Materials and structures 4, 693-718.

Griffith, D.T., Ashwill, T.D., 2011. The sandia 100-meter all-glass baseline wind turbine blade: Snl100-00. Sandia National Laboratories, Albuquerque, Report No. SAND2011-3779 .

Hegemier, G., Nair, S., 1977. A nonlinear dynamical theory for heterogeneous, anisotropic, elasticrods. AIAA Journal 15, 8-15.

Hodges, D.H., 1990. A mixed variational formulation based on exact intrinsic equations for dynamics of moving beams. International Journal of Solids and Structures 26, 1253-1273.

Hodges, D.H., 2003. Geometrically exact, intrinsic theory for dynamics of curved and twisted anisotropic beams. AIAA journal 41, 1131-1137.

Hodges, D.H., 2006. Nonlinear composite beam theory. AIAA, Reston, VA.

Ibrahimbegović, A., Mikdad, M.A., 1998. Finite rotations in dynamics of beams and implicit time-stepping schemes. International Journal for Numerical Methods in Engineering 41, 781-814.

Iura, M., Atluri, S.N., 1988. On a consistent theory, and variational formulation of finitely stretched and rotated 3-d spacecurved beams. Computational Mechanics 4, 73-88.

Jelenić, G., Crisfield, M.A., 1999. Geometrically exact 3d beam theory: implementation of a strain-invariant finite element for statics and dynamics. Computer Methods in Applied Mechanics and Engineering 171, 141-171.

Jeong, M.S., Kim, S.W., Lee, I., Yoo, S.J., Park, K.C., 2014. Investigation of wake effects on aeroelastic responses of horizontalaxis wind-turbines. AIAA Journal 52, 1133-1144.

Jonkman, J., Butterfield, S., Musial, W., Scott, G., 2009. Definition of a 5-mw reference wind turbine for offshore system development. National Renewable Energy Laboratory, Golden, CO, Technical Report No. NREL/TP-500-38060 .

Khaneh Masjedi, P., Ovesy, H.R., 2015. Chebyshev collocation method for static intrinsic equations of geometrically exact beams. International Journal of Solids and Structures 54, 183-191.

Khouli, F., Afagh, F.F., Langlois, R.G., 2009. Application of the first order generalized- $\alpha$ method to the solution of an intrinsic geometrically exact model of rotor blade systems. Journal of Computational and Nonlinear Dynamics $4,011006$.

Khouli, F., Griffiths, J., Afagh, F., Langlois, R., 2010. Actuation of slender thin-wall anisotropic open cross-section beams based on asymptotically correct vlasov theory. Journal of Intelligent Material Systems and Structures 21, 529-540.

Kim, H.S., Kim, J.S., 2013. A rankine-timonshenko-vlasov beam theory for anisotropic beams via an asymptotic strain energy transformation. European Journal of Mechanics-A/Solids 40, 131-138.

Kim, J.S., Cho, M., Smith, E.C., 2008. An asymptotic analysis of composite beams with kinematically corrected end effects. International Journal of Solids and Structures 45, 1954-1977.

Mäkinen, J., 2007. Total lagrangian reissner's geometrically exact beam element without singularities. International Journal for Numerical Methods in Engineering 70, 1009-1048.

Mason, J.C., Handscomb, D.C., 2003. Chebyshev polynomials. CRC Press LLC.

Meirovitch, L., 1997. Principles and Techniques of Vibrations. volume 1. Prentice Hall New Jersey.

Morandini, M., Chierichetti, M., Mantegazza, P., 2010. Characteristic behavior of prismatic anisotropic beam via generalized 
eigenvectors. International Journal of Solids and Structures 47, 1327-1337.

Pai, P.F., Nayfeh, A.H., 1994. A fully nonlinear theory of curved and twisted composite rotor blades accounting for warpings and three-dimensional stress effects. International Journal of Solids and Structures 31, 1309-1340.

Patil, M.J., Althoff, M., 2011. Energy-consistent, galerkin approach for the nonlinear dynamics of beams using intrinsic equations. Journal of Vibration and Control 17, 1748-1758.

Patil, M.J., Hodges, D.H., 2011. Variable-order finite elements for nonlinear, fully intrinsic beam equations. Journal of Mechanics of Materials and Structures 6, 479-493.

Patil, M.J., Hodges, D.H., Cesnik, C.E.S., 1999. Nonlinear aeroelasticity and flight dynamics of high-altitude long-endurance aircraft, in: 40th Structures, Structural Dynamics, and Materials Conference and Exhibit.

Petrov, E., Géradin, M., 1998. Finite element theory for curved and twisted beams based on exact solutions for three-dimensional solids part 1: Beam concept and geometrically exact nonlinear formulation. Computer Methods in Applied Mechanics and Engineering 165, 43-92.

Reissner, E., 1973. On one-dimensional large-displacement finite-strain beam theory. Studies in Applied Mathematics 52, $87-95$.

Resor, B.R., 2013. Definition of a $5 \mathrm{mw} / 61.5 \mathrm{~m}$ wind turbine blade reference model. Albuquerque, New Mexico, USA, Sandia National Laboratories, SAND2013-2569 2013.

Romero, I., Armero, F., 2002. An objective finite element approximation of the kinematics of geometrically exact rods and its use in the formulation of an energy-momentum conserving scheme in dynamics. International Journal for Numerical Methods in Engineering 54, 1683-1716.

Rosen, A., Loewy, R.G., Mathew, M.B., 1987. Use of twisted principal coordinates and nonphysical coordinates in blade analysis. Vertica 11, 541-572.

Schillinger, D., Evans, J.A., Reali, A., Scott, M.A., Hughes, T.J.R., 2013. Isogeometric collocation: Cost comparison with galerkin methods and extension to adaptive hierarchical nurbs discretizations. Computer Methods in Applied Mechanics and Engineering 267, 170-232.

Simo, J.C., 1985. A finite strain beam formulation. the three-dimensional dynamic problem. part i. Computer Methods in Applied Mechanics and Engineering 49, 55-70.

Simo, J.C., Vu-Quoc, L., 1988. On the dynamics in space of rods undergoing large motions a geometrically exact approach. Computer Methods in Applied Mechanics and Engineering 66, 125-161.

Simo, J.C., Vu-Quoc, L., 1991. A geometrically-exact rod model incorporating shear and torsion-warping deformation. International Journal of Solids and Structures 27, 371-393.

Tornabene, F., Fantuzzi, N., Ubertini, F., Viola, E., 2015. Strong formulation finite element method based on differential quadrature: a survey. Applied Mechanics Reviews 67, 020801.

Traugott, J.P., Patil, M.J., Holzapfel, F., 2006. Nonlinear modeling of integrally actuated beams. Aerospace Science and Technology 10, 509-518.

Yeo, H., Truong, K.V., Ormiston, R.A., 2014. Comparison of one-dimensional and three-dimensional structural dynamics modeling of advanced geometry blades. Journal of Aircraft 51, 226-235.

Yu, W., Hodges, D.H., Ho, J.C., 2012. Variational asymptotic beam sectional analysis-an updated version. International Journal of Engineering Science 59, 40-64.

Yu, W., Hodges, D.H., Volovoi, V.V., Fuchs, E.D., 2005. A generalized vlasov theory for composite beams. Thin-Walled Structures 43, 1493-1511. 


\section{AppendixA. Boundary Conditions}

Various boundary conditions associated with the free vibration of a non-rotating beam are depicted in Table A.14

Table A.14: Various Boundary Conditions for Non-Rotating Beams

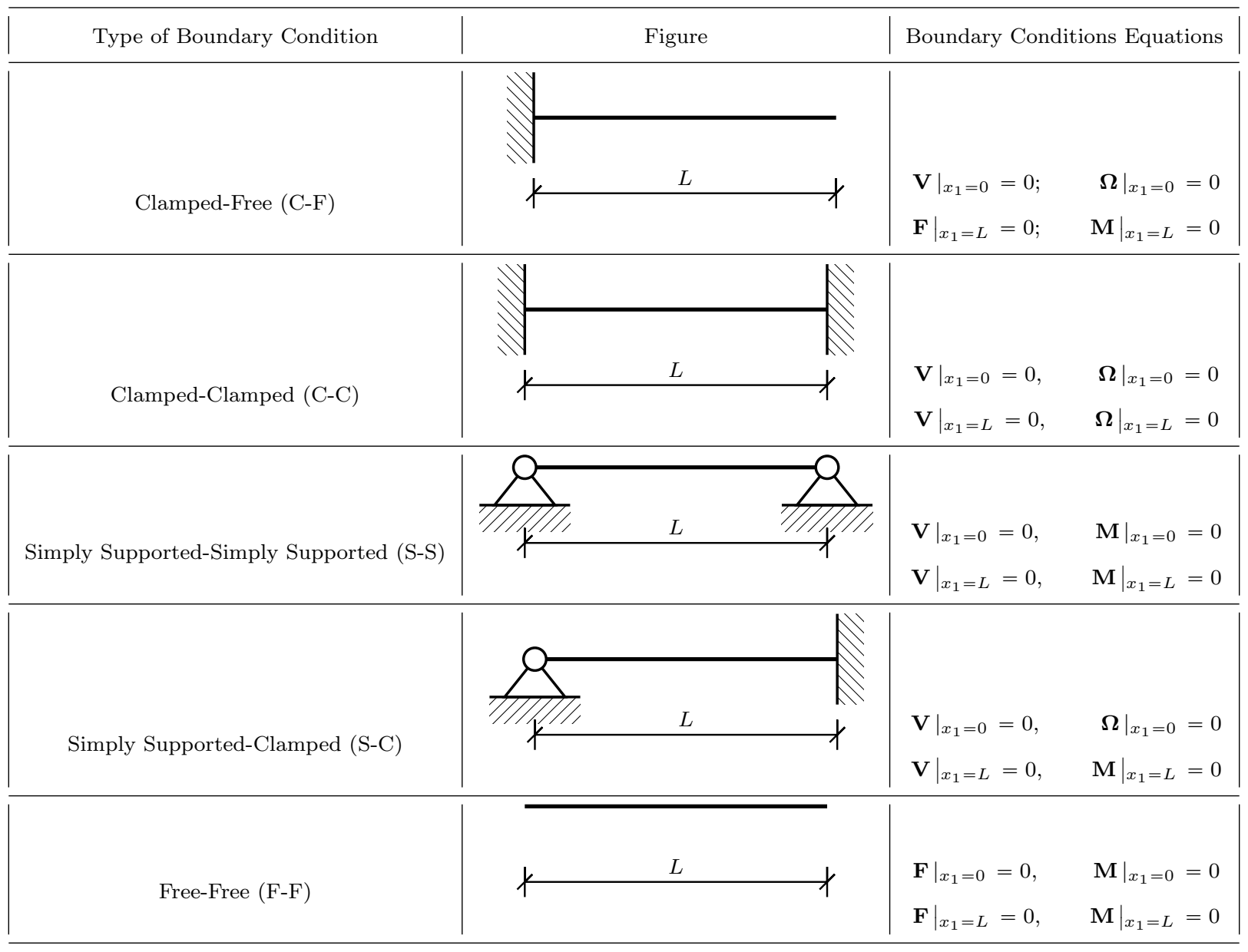

\section{AppendixB. Entries of $\mathcal{A}$ and $\mathcal{B}$ Matrices}

$$
\begin{aligned}
& \mathcal{A}_{11}=\phi_{F, 1}+\widetilde{\mathbf{K}}_{0} \cdot \phi_{F}-\widetilde{\mathbf{F}}_{0} \cdot Z^{T} \cdot \phi_{F} \\
\mathcal{A}_{12} & =-\widetilde{\mathbf{F}}_{0} \cdot T \cdot \phi_{M} \\
\mathcal{A}_{13} & =-\widetilde{\boldsymbol{\Omega}}_{0} \cdot \mu \Delta \cdot \phi_{V} \\
\mathcal{A}_{14} & =\widetilde{\Omega}_{0} \cdot \mu \widetilde{\bar{x}} \cdot \phi_{\Omega}+\widetilde{\mathbf{L}}_{0} \cdot \phi_{\Omega} \\
\mathcal{A}_{21} & =\left(\widetilde{\mathbf{e}}_{1}+\widetilde{\gamma}_{0}\right) \cdot \phi_{F}-\widetilde{\mathbf{M}}_{0} \cdot Z^{T} \cdot \phi_{F}-\widetilde{\mathbf{F}}_{0} \cdot R \cdot \phi_{F}
\end{aligned}
$$




$$
\begin{aligned}
& \mathcal{A}_{22}=\phi_{M, 1}+\widetilde{\mathbf{K}}_{0} \cdot \phi_{M}-\widetilde{\mathbf{M}}_{0} \cdot T \cdot \phi_{M}-\widetilde{\mathbf{F}}_{0} \cdot Z \cdot \phi_{M} \\
& \mathcal{A}_{23}=\widetilde{\mathbf{L}}_{0} \cdot \phi_{V}-\widetilde{\mathbf{\Omega}}_{0} \cdot \mu \tilde{\bar{x}} \cdot \phi_{V}-\tilde{\mathbf{V}}_{0} \cdot \mu \Delta \cdot \phi_{V} \\
& \mathcal{A}_{24}=\widetilde{\mathbf{P}}_{0} \cdot \phi_{\Omega}+\widetilde{\mathbf{V}}_{0} \cdot \mu \tilde{\bar{x}} \cdot \phi_{\Omega}-\widetilde{\Omega}_{0} \cdot I \cdot \phi_{\Omega} \\
& \mathcal{A}_{31}=-\tilde{\mathbf{V}}_{0} \cdot Z^{T} \cdot \phi_{F}-\tilde{\Omega}_{0} \cdot R \cdot \phi_{F} \\
& \mathcal{A}_{32}=-\tilde{\mathbf{V}}_{0} \cdot T \cdot \phi_{M}-\widetilde{\Omega}_{0} \cdot Z \cdot \phi_{M} \\
& \mathcal{A}_{33}=\phi_{V, 1}+\widetilde{\mathbf{K}}_{0} \cdot \phi_{V} \\
& \mathcal{A}_{34}=\left(\widetilde{\mathbf{e}}_{1}+\widetilde{\gamma}_{0}\right) \cdot \phi_{\Omega} \\
& \mathcal{A}_{41}=-\widetilde{\Omega}_{0} \cdot Z^{T} \cdot \phi_{F} \\
& \mathcal{A}_{42}=-\widetilde{\boldsymbol{\Omega}}_{0} \cdot T \cdot \phi_{M} \\
& \mathcal{A}_{44}=\phi_{\Omega, 1}+\widetilde{\mathbf{K}}_{0} \cdot \phi_{\Omega} \\
& \mathcal{B}_{13}=\mu \Delta . \phi_{V} \\
& \mathcal{B}_{14}=-\mu \tilde{\bar{x}} . \phi_{\Omega} \\
& \mathcal{B}_{23}=\mu \tilde{\bar{x}} . \phi_{V} \\
& \mathcal{B}_{24}=I . \phi_{\Omega} \\
& \mathcal{B}_{31}=R . \phi_{F} \\
& \mathcal{B}_{32}=Z . \phi_{M} \\
& \mathcal{B}_{41}=Z^{T} . \phi_{F} \\
& \mathcal{B}_{42}=T . \phi_{M}
\end{aligned}
$$

\section{AppendixC. Chebyshev Polynomials}

The Chebyshev polynomials are a sequence of orthogonal polynomials. The Chebyshev polynomial $T_{n}(x)$ of the first kind is a polynomial in $x$ of degree $n$ and is defined as (Mason and Handscomb (2003)):

$$
T_{n}(x)=\cos (n \theta) \quad \text { when } \quad x=\cos \theta, \quad-1 \leqslant x \leqslant+1
$$

Combining the trigonometric identity;

$$
\cos (n \theta)+\cos ((n-2) \theta)=2 \cos \theta \cos (n-1) \theta
$$

with Eq. C.1, the recurrence relation can be obtained as:

$$
\begin{aligned}
& T_{n}(x)=2 x T_{n-1}(x)-T_{n-2}(x), \quad n=2,3, \ldots, \\
& \text { where } \\
& T_{0}(x)=1, T_{1}(x)=x
\end{aligned}
$$


The first few Chebyshev polynomials of the first kind are:

$$
\begin{aligned}
& T_{2}(x)=2 x^{2}-1 \\
& T_{3}(x)=4 x^{3}-3 x \\
& T_{4}(x)=8 x^{4}-8 x^{2}+1 \\
& T_{5}(x)=16 x^{5}-20 x^{3}+5 x
\end{aligned}
$$

Using the weight function $w=\left(1-x^{2}\right)^{-\frac{1}{2}}$ one can find that the Chebyshev polynomials of the first kind satisfy the orthogonality condition:

$$
\int_{-1}^{1} \frac{T_{i}(x) T_{j}(x)}{\sqrt{1-x^{2}}}=\left\{\begin{array}{lr}
0, & i \neq j \\
\frac{\pi}{2}, & i=j \neq 0 \\
\pi, & i=j=0
\end{array}\right.
$$

It is noted that for any arbitrary range $a \leqslant x \leqslant b$ the Chebyshev polynomials can be shifted by replacing the independent variable $x$ in Eq. C.4 by:

$$
x=\frac{2}{b-a} x-\frac{b+a}{b-a}
$$

